Linköping Studies in Science and Technology

Dissertation No. 2190

Summation-by-parts

formulations for

flow problems

Fredrik Laurén 

Linköping Studies in Science and Technology.

Dissertations No. 2190

\section{Summation-by-parts formulations for flow problems}

Fredrik Laurén

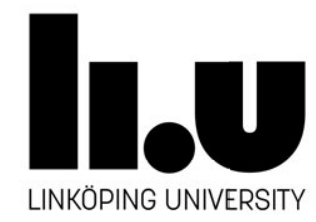

Department of Mathematics

Linköping, 2022 
(c) EY This work is licensed under a Creative Commons Attribution 4.0 International License.

https://creativecommons.org/licenses/by/4.0/

Linköping Studies in Science and Technology. Dissertations No. 2190

Summation-by-parts formulations for flow problems

Copyright (C) Fredrik Laurén, 2022

Department of Mathematics

Linköping University

SE-581 83 Linköping, Sweden

Email: fredrik.lauren@liu.se

ISSN 0345-7524

ISBN 978-91-7929-121-1 (Print) ISBN 978-91-7929-122-8 (PDF)

Printed by LiU-Tryck, Linköping, Sweden, 2022 


\section{Abstract}

Many problems in engineering and physics can be described by partial differential equations (PDEs). Augmented with proper initial and boundary conditions, the PDE forms an initial-boundary value problem (IBVP). An IBVP is said to be well-posed if a unique solution bounded by the given data exists. The behavior of the solution as well as the well-posedness of the IBVP are heavily influenced by the form and position of the boundary conditions. The solution, if it exists, to an IBVP cannot in general be obtained in closed form. Instead, one can compute an approximation using numerical methods. Summation-by-parts (SBP) operators together with the simultaneous approximation term (SAT) technique can be used to form stable numerical methods that generate accurate approximations. All discretizations in this thesis are based on the SBP-SAT framework.

The first part of the thesis concentrates on IBVPs describing fluid motion. Different sets of boundary conditions are investigated in terms of energy-boundedness and spectral properties. Wall models are also studied and used to aid coarse-grid simulations in an energy stable manner.

In the second part of the thesis, special discretization operators are derived. First, single-block SBP operators are combined with interpolation operators. The result is a single SBP operators on a multi-block grid that encapsulates both the metric terms and the interface treatments. Second, new SBP operators are developed for an application in high-energy physics. The new operators preserves the unit-trace, which is important since it enables a probability interpretation of the density matrix. 


\section{Sammanfattning på svenska}

Många fenomen inom ingenjörsvetenskapen och fysiken kan beskrivas med partiella differentialekvationer (PDE:er). Tillsammans med lämpliga initial - och randvillkor bildar PDE:en ett initial-randvärdesproblem (IRVP) som sägs vara välställt om en unik lösning existerar som är begränsad av den givna datan. Hur lösningen beter sig, samt om IRVP:et är välställt eller inte, beror till stor del både på formen och position av randvillkoren. Även om en unik lösning existerar till ett givet IRVP, är det inte troligt att den går att uttrycka på sluten form. Istället försöker man få fram dess approximation med hjälp av numeriska metoder. SBP operatorer (SBP från engelskans summation-by-parts) kan tillsammans med SATtekniken (SAT från engelskans simultaneous approximation term) användas till att konstruera stabila numeriska algoritmer som ger noggranna approximationer. Samtliga diskretiseringar i denna avhandling använder sig av SBP-SAT-konceptet.

Den första delen av avhandlingen, som består av Artikel I, II, III och IV, handlar om IRVP som beskriver rörelser hos en fluid. Speciellt läggs fokus på olika randvillkor som används vid öppna ränder. Spektrala egenskaper och energistabilitet undersöks med hjälp av normalmodsanalys och energimetoden. Väggmodeller, som används vid turbulenta simuleringar på grova nät, undersöks också.

I den andra delen av avhandlingen utvecklas speciella SBP operatorer. I Artikel V kombineras SBP operatorer definierade för ett block med interpolationsoperatorer. Resultatet är en diskretisering som innehåller både de metriska termerna och hanteringen av gränssnitteten mellan de olika blocken. I Artikel VI utvecklas en ny invariansbevarande diskretisering för Lindblads ekvationer som används inom högenergifysik. 


\section{Acknowledgments}

Above all, I would like to thank my supervisor Jan Nordström for his incredible level of commitment and support during my time at LiU. You have from my first day given me challenging and inspiring projects but also an enormous amount of feedback and suggestions to move forward.

To my co-supervisor Andrew Winters. Thank you for all the support, inspiring discussions and also for showing cool simulations.

I would also like to thank all of my colleagues at MAI for their day-to-day support and positive energy, especially former and current PhD-students.

I could never have wished for a better FRIEND (and colleague) to share an office with for my first four years at LiU. Thank you Oskar for all the good days and fun memories. Roghi, I am so happy for all time we spent both at MAI and outside of the university. Thank you for being an amazing friend and for letting me practice my drawing skills on your whiteboard.

To my friends outside of MAI, thank you for making these years more fun with climbing, hiking, floorball, dinners, bbq, and lots more! I would also like to thank my family for always believing in me. I am forever grateful and truly feel lucky for your support. To Matilda, you are the most amazing person I know and you have encouraged me during many late nights and busy weekends. I love you. 


\section{List of papers}

I Laurén, Fredrik, and Jan Nordström. "Practical inlet boundary conditions for internal flow calculations." Computers and Fluids 175 (2018): 159-166.

II Nordström, Jan, and Fredrik Laurén. "The spatial operator in the incompressible Navier-Stokes, Oseen and Stokes equations." Computer Methods in Applied Mechanics and Engineering 363 (2020):112857.

III Laurén, Fredrik, and Jan Nordström. "Spectral properties of the incompressible Navier-Stokes equations." Journal of Computational Physics 429 (2021):110019

IV Laurén, Fredrik, and Jan Nordström. "Energy stable wall modeling for the Navier-Stokes equations." LiTH-MAT-R-2021/04-SE. Submitted for publication (2021).

V Lundquist, Tomas, Fredrik Laurén and Jan Nordström. "A multi-domain summation-by-parts formulation for complex geometries." LiTH-MAT-R2021/05-SE. Submitted for publication (2021).

VI Ålund, Oskar, Yukinao Akamatsu, Fredrik Laurén, Takahiro Miura, Jan Nordström, and Alexander Rothkopf. "Trace preserving quantum dynamics using a novel reparametrization-neutral summation-by-parts difference operator." Journal of Computational Physics 425 (2021):109917.

I have contributed to papers I-IV by deriving most of the theoretical results as well as performing all the numerical computations. The manuscripts were written by me, but with generous editorial support from my supervisor Jan Nordström. The main result of paper $\mathrm{V}$ is derived by the first author, Tomas Lundquist. I contributed to paper $\mathrm{V}$ by writing parts of the manuscript and by developing the numerical scheme used for the numerical experiments. In paper VI, I developed the spatial discretization in collaboration with Oskar Ålund and Jan Nordström. 


\section{Contents}

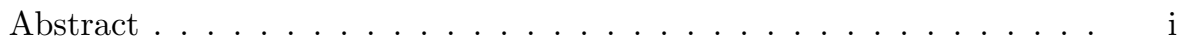

Sammanfattning på svenska . . . . . . . . . . . . . . iii

Acknowledgments . . . . . . . . . . . . . . . . . . V

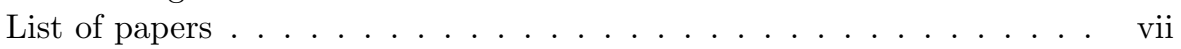

Contents ............................ ix

1 Introduction 1

2 Initial-boundary value problems and the energy method 3

2.1 The energy method and well-posedness . . . . . . . . . . . . 4

2.2 Weak boundary conditions . . . . . . . . . . . . . 5

3 Summation-by-parts operators $\quad 7$

3.1 Simulations approximation terms and stability . . . . . . . . 9

3.2 SBP operators in higher dimensions . . . . . . . . . . . . . 10

4 The incompressible Navier-Stokes equations 13

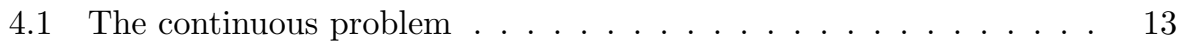

4.2 The discrete problem . . . . . . . . . . . . . . . . . . . . 15

4.3 Numerical experiments . . . . . . . . . . . . . . 15

5 Spectral analysis $\quad 19$

5.1 Fourier analysis . . . . . . . . . . . . . . . . . . 20

5.2 Normal mode analysis . . . . . . . . . . . . . . . . . 20

5.3 The discrete spectrum . . . . . . . . . . . . . . . . . . . . . 22

5.4 The convergence rate to steady state . . . . . . . . . . . . 22

6 Summary of articles $\quad 25$

6.1 Article I . . . . . . . . . . . . . . . . . . . . . . 25

6.2 Article II . . . . . . . . . . . . . . . . . . . . . . . . . . . . . . . . . . . . . . 25

6.3 Article III . . . . . . . . . . . . . . . . . . 26

6.4 Article IV . . . . . . . . . . . . . . . . . . . . . . . . . . . . . . . . . 27

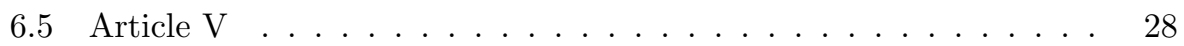

6.6 Article VI . . . . . . . . . . . . . . . . . 30

$\begin{array}{ll}\text { References } & 31\end{array}$

$\begin{array}{ll}\text { Article I } & 35\end{array}$

Article II $\quad 45$

$\begin{array}{lr}\text { Article III } & 69\end{array}$

$\begin{array}{ll}\text { Article IV } & 93\end{array}$ 
Article V

Article VI

135 


\section{Chapter 1}

\section{Introduction}

Many problems in engineering and natural science can be described by partial differential equations (PDEs) [47]. Although the underlying PDEs might be easy to formulate for a physical phenomena, a closed form mathematical expression of the solution is seldom easy to obtain. Instead, scientific computing tools can be used, where the target is a numerical approximation of the solution. The process of obtaining the numerical solution by using computers is known as the numerical method [43].

When relying on numerical methods, the concept of well-posedness becomes important [8]. A well-posed continuous mathematical problem (or a physical phenomena) has a unique solution that changes continuously with the input data. On the discrete side, it is required that the numerical scheme is stable [48, 17]. A stable numerical method that approximates a well-posed linear continuous problem will produce accurate approximations on a fine grid [48]. It is therefore reasonable to require the underlying continuous model to be well-posed before any numerical method is considered. The converse is an ill-posed problem. An ill-posed problem must be regularized, i.e. transformed into a well-posed problem, before it can be solved [42].

To investigate well-posedness, the energy method can be employed [17]. A key concept in this investigation strategy is integration-by-parts (IBP). In order to mimic the continuous energy analysis, difference operators on summation-byparts (SBP) form can be used [16]. These operators satisfy the discrete version of IBP and can, together with weakly imposed boundary conditions via the simultaneous approximation term (SAT) technique [6], generate stable schemes. The SBP-SAT framework covers many forms of numerical approximation techniques such as finite-difference, continuous and discontinuous Galerkin and finite-volume methods $[49,11]$.

The energy method and the SBP-SAT framework are the core building blocks of the work in this thesis, which consists of six articles. The major focus (Articles I-IV) is on PDEs describing fluid motion (i.e. the Euler and the Navier-Stokes equations) $[50,40]$. These PDEs are usually posed on restricted domains where boundary conditions must be provided leading to an initial-boundary value problem (IBVP). In Article I, the compressible Euler equations together with a set of 
boundary conditions used for internal flow computations are considered. The influence of boundary conditions on the spatial operator of the incompressible Navier-Stokes (INS) equations are investigated in Article II. In Article III, several sets of open boundary conditions to the INS equations are compared. How to use wall models together with the INS equations in an energy stable manner is the content of Article IV.

Next, the focus is shifted to the derivation of special SBP operators. In Article $\mathrm{V}$, a multi-block SBP formulation is formed. Trace-preserving SBP operators for the Lindblad equations, which describes the evolution of dissipative quantum systems, are derived in Article VI.

The upcoming sections introduce IBVPs, the SBP-SAT framework, the INS equations and the normal mode analysis. After that, each article is summarized. 


\section{Chapter 2}

\section{Initial-boundary value problems and the energy method}

For many applications in engineering and science the governing physical principles can be formulated as IBVPs [22]. In this section, a one-dimensional transport problem [8] is presented and formulated as an IBVP. This is done for the purposes of presentation and introducing the topics of well-posedness, the energy method, and weak boundary conditions. The concepts introduced in this section are used in Articles I-V.

Suppose we are interested in the position of a one-dimensional pulse traveling on a long thin string. The position of the pulse is denoted by $u$ and it is assumed to be unaffected by external forces. Further, the pulse moves at a constant (unit) speed along the string in the positive $x$-direction. To determine the position of the pulse at a future time, its initial state must be known. This situation can be described by a PDE equipped with an initial condition, resulting in the initial-value problem (IVP) [47]

$$
\begin{aligned}
u_{t}+u_{x} & =0, & & t>0, \\
u(0, x) & =f(x), & & t=0 .
\end{aligned}
$$

The unique solution to $(2.1)$ is $u=u(t, x)=f(x-t)$. Hence, the pulse is transported at the constant (unit) speed in the positive $x$-direction, see Figure 2.

If a finite domain, say $x \in[0,1]$, is considered instead, specific boundary conditions must be supplied to (2.1), resulting in the following IBVP

$$
\begin{aligned}
u_{t}+u_{x}=0, & t>0, & x \in[0,1], \\
u(0, x)=f(x), & t=0, & x \in[0,1], \\
u(t, 0)=g(t), & t>0, & x=0 .
\end{aligned}
$$

The boundary condition is imposed at the left boundary, where new information enters the domain [48]. Without prescribing the position of the incoming position 


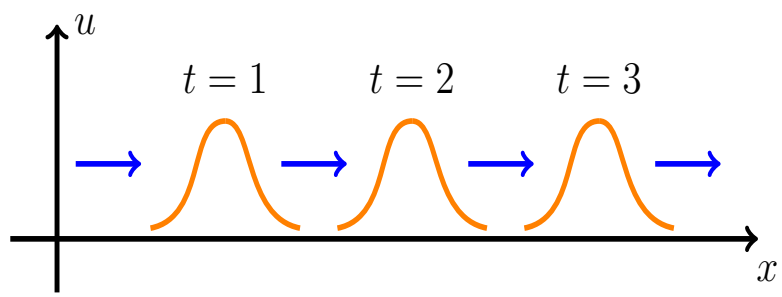

Figure 2.1: The pulse traveling at constant speed.

of the pulse, its behavior at later times cannot be determined. Hence, to obtain a proper description of the pulse in the domain, it is necessary to know

i) The governing equation, $u_{t}+u_{x}=0$.

ii) The initial condition, $u(0, x)=f(x)$.

iii) The boundary condition, $u(t, 0)=g(t)$ imposed at $x=0$.

\subsection{The energy method and well-posedness}

Generalizing (2.2), the linear IBVP becomes

$$
\begin{aligned}
& u_{t}+\mathcal{D} u=F, \quad t>0, \quad x \in \Omega, \\
& u=f(x), \quad t=0, \quad x \in \Omega, \\
& \mathcal{B} u=g(t), \quad t>0, \quad x \in \partial \Omega .
\end{aligned}
$$

In (2.3), $\mathcal{D}$ is a differential operator in space defined on the domain $\Omega$ and $\mathcal{B}$ is a boundary operator that acts on the solution at $\partial \Omega$, the boundary of $\Omega[16]$.

For two functions $u, v \in L^{2}(\Omega)$, let

$$
\langle u, v\rangle=\int_{\Omega} u v \mathrm{~d} x, \quad\|u\|^{2}=\langle u, u\rangle
$$

denote the scalar product and the corresponding norm. To properly describe $u$ as was done with the pulse in the previous section, (2.3) must be well-posed.

Definition 1. The $\operatorname{IBVP}(2.3)$ is well-posed if for $F=0$ and $g=0$ (i.e. no forcing function and homogeneous boundary conditions) a unique solution satisfying

$$
\|u\|^{2} \leq K e^{\alpha t}\|f\|^{2}
$$

exists. In (2.4), $K$ and $\alpha$ are independent of $f$. It is said to be strongly well-posed if the unique solution satisfy

$$
\|u\|^{2} \leq K e^{\alpha t}\left(\|f\|^{2}+\int_{0}^{t}\|F\|^{2}+|g|^{2} \mathrm{~d} \tau\right),
$$

where $K$ and $\alpha$ are independent of $f, F$ and $g$. [16] 
The estimate (2.5) implies that the solution to (2.3) is bounded by the data $F, f$ and $g$. A small perturbation in the data, will cause a small perturbation in the solution (as long as $K$ and $\alpha$ are of moderate size) [16]. For a linear operator $\mathcal{D}$, existence is related to correctly specifying the boundary conditions and can by investigated by using the Laplace-transform technique [17]. Uniqueness follows directly from the energy estimates in Definition 1. A solution that satisfies (2.4) or (2.5) is henceforth referred to as energy bounded (even if the PDE is nonlinear). In Article I, the linearized Euler equations are proven to be well-posed, while in Articles II-IV, energy boundedness of the INS equations are investigated.

To investigate energy boundedness, the energy method $[17,16]$ can be used. Multiplying the first equation in $(2.2)$ by $2 u$ and integrating over the spatial domain yields

$$
2\left\langle u, u_{t}\right\rangle=2\left\langle u,-u_{x}\right\rangle=-\left.u^{2}\right|_{0} ^{1}=g(t)^{2}-u(t, 1)^{2} \leq g(t)^{2} .
$$

Integrating (2.6) in time, and observing that $\frac{d}{d t}\|u\|^{2}=2\left\langle u, u_{t}\right\rangle$, leads to

$$
\|u\|^{2} \leq\|f\|^{2}+\int_{0}^{t} g^{2} \mathrm{~d} \tau,
$$

where $K=1$ and $\alpha=0$ in (2.5). Hence, (2.2) is strongly well-posed. The energy method can be summarized as follows

i) Multiply the governing equations by the solution and integrate in space.

ii) Note that $\frac{d}{d t}\|u\|^{2}=2\left\langle u, u_{t}\right\rangle$.

iii) Substitute $u_{t}$ according to the governing equations of the IBVP.

iv) Integrate by parts to separate boundary and volume contributions.

v) Impose boundary conditions to limit the boundary terms.

vi) Integrate in time.

Remark 1. For IBVP in higher dimensions, the boundary terms in step iv) come in the form of a boundary integral. If higher derivatives are included in the governing equations, dissipative volume integrals could also remain after IBP [8].

\subsection{Weak boundary conditions}

An alternative formulation of (2.3) is obtained by imposing the boundary condition weakly via a penalty term [30]. This procedure simplifies the derivation of the penalty parameters of the discrete scheme and is used in Articles II-IV. For (2.2), the magnitude of this penalty term increases in proportion to the magnitude of $u(t, 0)-g(t)$ (the deviation from the boundary condition). In essence, the penalty term "forces" the solution to conform with the boundary condition at the boundary. To illustrate this, (2.2) is reformulated to be

$$
\begin{aligned}
& u_{t}+u_{x}=\sigma \mathcal{L}(u-g), \quad t>0, \quad x \in[0,1], \\
& u(0, x)=f(x), \quad t=0, \quad x \in[0,1] \text {. }
\end{aligned}
$$


In $(2.7), \mathcal{L}$ is a lifting operator $[2,30]$, which is chosen such that

$$
\langle v, \mathcal{L}(u)\rangle=\left.u v\right|_{x=0} .
$$

The parameter $\sigma$ is at this point unknown but will be determined so that an energy estimate is obtained. Applying the energy method to (2.7) yields

$$
\frac{d}{d t}\|u\|^{2}=u(t, 0)^{2}-u(t, 1)^{2}+2 \sigma u(t, 0)[u(t, 0)-g(t)] .
$$

The choice $\sigma \leq-1 / 2$ leads to an energy estimate of the form (2.4) for $g(t)=0$. The specific choice $\sigma=-1$ results in

$$
\frac{d}{d t}\|u\|^{2}=-u(t, 1)^{2}-[u(t, 0)-g(t)]^{2}+g(t)^{2} \leq g(t)^{2},
$$

which is identical to $(2.6)$, but with the additional damping terms $-[u(t, 0)-g(t)]^{2}$.

By weakly imposing the boundary condition, the continuous problem can be analyzed without considering any numerical method. Furthermore, all discretization techniques on SBP-SAT form will be able to mimic the continuous analysis. 


\section{Chapter 3}

\section{Summation-by-parts operators}

As noted in Section 2.1, integration-by-parts is the key property for the energy method. In the discrete setting, a similar property is desirable in order to obtain a stable numerical approximation of an IBVP. By design, SBP operators possess exactly that property on a discrete level. These operators were first derived in $[24,23]$. Later, the framework has been extended to cover a broad family of numerical methods, see for example [33, 5, 13]. The numerical schemes in Articles I-VI are constructed using the SBP-SAT framework and all difference operators used to approximate partial derivatives satisfy Definition 2 (given below). An extended definition (with some stability issues [37]) can be found in [10].

Definition 2. The matrix $D=P^{-1} Q$ is an $S B P$ operator of order $k$ if

1. $D \boldsymbol{x}^{m}=m \boldsymbol{x}^{m-1}, \quad m=0, \ldots, k$.

2. $P$ is symmetric and positive definite.

3. $Q+Q^{\top}=B=\operatorname{diag}(-1,0, \ldots, 0,1)$.

In the definition above, $\boldsymbol{x}^{m}=\left(x_{0}^{m}, \ldots, x_{N}^{m}\right)$ is the element-wise exponentiation.

In order to obtain stable numerical approximations for variable coefficient and nonlinear problems, $P$ must be diagonal so that it commutes with general diagonal matrices $[49,29]$.

To demonstrate the SBP property, the first order finite-difference SBP operator will be used. Details on high-order operators are given in [16]. To start, the interval $\Omega=[0,1]$ is discretized by using $N+1$ equidistant points: $x_{i}=i h, i=$ $0 \ldots N, h=1 / N$. The scalar function $f(x)$ defined on $\Omega$ is represented on the grid by $\boldsymbol{f}=\left(f\left(x_{0}\right), \ldots f\left(x_{N}\right)\right)^{\top}$. A simple technique to approximate $\boldsymbol{f}^{\prime}$ is with central finite differences in the interior and appropriate forward/backward stencils at the 
boundaries. These operations can be represented by the matrix

$$
D=\frac{1}{h}\left(\begin{array}{ccccc}
-1 & 1 & & & \\
-\frac{1}{2} & 0 & \frac{1}{2} & & \\
& \ddots & \ddots & \ddots & \\
& & -\frac{1}{2} & 0 & \frac{1}{2} \\
& & & -1 & 1
\end{array}\right)
$$

which leads to

$$
D \boldsymbol{f}=\left(\begin{array}{c}
f^{\prime}\left(x_{0}\right) \\
f^{\prime}\left(x_{1}\right) \\
\vdots \\
f^{\prime}\left(x_{N-1}\right) \\
f^{\prime}\left(x_{N}\right)
\end{array}\right)+\left(\begin{array}{c}
\mathcal{O}(h) \\
\mathcal{O}\left(h^{2}\right) \\
\vdots \\
\mathcal{O}\left(h^{2}\right) \\
\mathcal{O}(h)
\end{array}\right) \approx \boldsymbol{f}^{\prime}
$$

The differentiation matrix can be factored into two matrices as $D=P^{-1} Q$, where

$$
P=h\left(\begin{array}{ccccc}
\frac{1}{2} & & & & \\
& 1 & & & \\
& & \ddots & & \\
& & & 1 & \\
& & & & \frac{1}{2}
\end{array}\right), Q=\frac{1}{2}\left(\begin{array}{ccccc}
-1 & 1 & & & \\
-1 & 0 & 1 & & \\
& \ddots & \ddots & \ddots & \\
& & -1 & 0 & 1 \\
& & & -1 & 1
\end{array}\right) \text {. }
$$

The quadrature matrix $P$ contains the weights of the second-order composite Trapezoidal rule. It is symmetric and positive definite so that it defines the discrete scalar product $\langle\boldsymbol{v}, \boldsymbol{u}\rangle_{P}=\boldsymbol{v}^{\top} P \boldsymbol{u}$ and the discrete norm $\|\boldsymbol{f}\|_{P}=\sqrt{\boldsymbol{f}^{\top} P \boldsymbol{f}} \approx\|f\|_{L^{2}}$. The stencil matrix $Q$ is almost skew-symmetric since

$$
Q+Q^{\top}=\operatorname{diag}(-1,0, \ldots, 0,1)=B .
$$

The separation of $D$ into $P^{-1}$ and $Q$ is motivated by

$$
\begin{aligned}
\langle\boldsymbol{v}, D \boldsymbol{u}\rangle_{P}+\langle D \boldsymbol{v}, \boldsymbol{u}\rangle_{P} & =\boldsymbol{v}^{\top} P D \boldsymbol{u}+(D \boldsymbol{v})^{\top} P \boldsymbol{u} \\
& =\boldsymbol{v}^{\top} P\left(P^{-1} Q\right) \boldsymbol{u}+\boldsymbol{v}^{\top}\left(Q^{\top} P^{-1}\right) P \boldsymbol{u} \\
& =\boldsymbol{v}^{\top}\left(Q+Q^{\top}\right) \boldsymbol{u} \\
& =\boldsymbol{v}^{\top} B \boldsymbol{u} \\
& =u_{N} v_{N}-u_{0} v_{0},
\end{aligned}
$$

which discretely mimics the integration-by-parts formula

$$
\left\langle v, u^{\prime}\right\rangle+\left\langle v^{\prime}, u\right\rangle=\int_{0}^{1} v u^{\prime} \mathrm{d} x+\int_{0}^{1} v^{\prime} u \mathrm{~d} x=\left.u v\right|_{x=1}-\left.u v\right|_{x=0} .
$$

Returning to the advection equation in (2.2). The derivative matrix $D$ is now used to approximate $\boldsymbol{u}_{x}$, which yields the semi-discrete advection equation

$$
\boldsymbol{u}_{t}+D \boldsymbol{u}=0 .
$$


In the continuous setting, the governing equation were multiplied by $2 u$ followed by an integration. The corresponding discrete operations are obtained by multiplying (3.1) by $2 \boldsymbol{u}^{\top} P$ from the left, where the multiplication of the quadrature matrix $P$ mimics the continuous integration step. The multiplication leads to

$$
\begin{aligned}
2\left\langle\boldsymbol{u}, \boldsymbol{u}_{t}\right\rangle_{P} & =2 \boldsymbol{u}^{\top} P \boldsymbol{u}_{t}=-2 \boldsymbol{u}^{\top} P D \boldsymbol{u} \\
& =-\boldsymbol{u}^{\top} P D \boldsymbol{u}-\left(\boldsymbol{u}^{\top} P D \boldsymbol{u}\right)^{\top} \\
& =-\boldsymbol{u}^{\top}\left(P D+D^{\top} P\right) \boldsymbol{u} \\
& =-\boldsymbol{u}^{\top}\left(Q+Q^{\top}\right) \boldsymbol{u} \\
& =-\boldsymbol{u}^{\top} B \boldsymbol{u}=-u_{N}^{2}+u_{0}^{2} .
\end{aligned}
$$

By studying the last row of (3.2), we realize that boundary conditions must be imposed in a correct way in order to obtain an energy bounded approximation.

\subsection{Simulations approximation terms and stability}

The SAT technique [6] is used to impose boundary conditions in Articles I-V and is demonstrated on the advection equation below. Similar to the formulation presented in Section 2.2, the boundary condition is weakly imposed via a penalty term. Discretizing (2.7) in space results in the IVP

$$
\begin{aligned}
\boldsymbol{u}_{t}+D \boldsymbol{u} & =\sigma \mathcal{L}(\boldsymbol{u}-\boldsymbol{g}), & & t>0, \\
\boldsymbol{u} & =\boldsymbol{f}, & & t=0 .
\end{aligned}
$$

In $(3.3), \boldsymbol{g}=(g(t), 0 \ldots, 0)^{\top}$ contains the boundary data, and the right-hand side is a penalty term that forces (pulls) $u_{0}$ towards $g(t)$. The better the boundary condition is approximated, the smaller the magnitude of the penalty term becomes. If $u_{0}=g(t)$, then the penalty term vanishes and what remains is only the approximation to the original PDE with the correct boundary terms. Using the SAT technique, the PDE and the boundary conditions are simultaneously approximated.

The operator $\mathcal{L}=P^{-1} E_{0}$, with $E_{0}=\operatorname{diag}(1,0, \ldots, 0)$, is the discrete analogue of the lifting operator introduced in (2.8). It positions the boundary condition correctly since

$$
\langle\boldsymbol{v}, \mathcal{L} \boldsymbol{u}\rangle_{P}=\boldsymbol{v}^{\top} P P^{-1} E_{0} \boldsymbol{u}=u_{0} v_{0}
$$

The parameter $\sigma$ is at this stage not known and will be determined so that (3.3) is stable, which is introduced next.

Definition 3. The semi-discrete problem (3.3) is stable if for $g(t)=0$

$$
\|\boldsymbol{u}\|_{P}^{2} \leq K e^{\alpha_{d} t}\|\boldsymbol{f}\|_{P}^{2},
$$

where $K$ and $\alpha_{d}$ are constants independent of $\boldsymbol{f}$. It is said to be strongly stable if

$$
\|\boldsymbol{u}\|_{P}^{2} \leq K e^{\alpha_{d} t}\left(\|\boldsymbol{f}\|_{P}^{2}+\int_{0}^{t}|g|^{2} \mathrm{~d} \tau\right)
$$

where $K$ and $\alpha_{d}$ are constants independent of $\boldsymbol{f}$ and $g .[16]$ 
In order to investigate stability, (3.3) is multiplied by $2 \boldsymbol{u}^{\top} P$, which yields (3.2) plus additional terms from the SAT:

$$
2\left\langle\boldsymbol{u}, \boldsymbol{u}_{t}\right\rangle_{P}=-2 \boldsymbol{u}^{\top} P D \boldsymbol{u}+2 \sigma \boldsymbol{u}^{\top} E_{0}(\boldsymbol{u}-\boldsymbol{g})=-u_{N}^{2}+u_{0}^{2}+2 \sigma u_{0}\left[u_{0}-g(t)\right] .
$$

Note that $\frac{d}{d t}\|\boldsymbol{u}\|_{P}^{2}=2\left\langle\boldsymbol{u}, \boldsymbol{u}_{t}\right\rangle_{P}$. As in Section 2.2 , the choice $\sigma=-1$ yields

$$
\frac{d}{d t}\|\boldsymbol{u}\|_{P}^{2}=-u_{N}^{2}-\left[u_{0}-g(t)\right]^{2}+g(t)^{2},
$$

which is the discrete equivalent of (2.9). Integrating in time yields

$$
\|\boldsymbol{u}\|_{P}^{2} \leq\|\boldsymbol{f}\|_{P}^{2}+\int_{0}^{t} g^{2} \mathrm{~d} \tau,
$$

where $K=1$ and $\alpha_{d}=0$ in (3.6). Hence, (3.3) is strongly stable with $\sigma=-1$. A stability bound of the form (3.5) is obtained for $\sigma \leq-1 / 2$.

The discrete energy method for SBP-SAT schemes, demonstrated above, can be summarized as follows

i) Multiply the governing equations by $2 \boldsymbol{u}^{\top} P$.

ii) Note that $\frac{d}{d t}\|\boldsymbol{u}\|_{P}^{2}=2\left\langle\boldsymbol{u}, \boldsymbol{u}_{t}\right\rangle_{P}$.

iii) Substitute $\boldsymbol{u}_{t}$ according to the governing equations of the IVP.

iv) Utilize the SBP property of the SBP operators.

v) Determine the penalty parameter $\sigma$ so that the boundary terms are limited.

vi) Integrate in time.

Once stability is obtained, (3.3) can be integrated in time using a standard numerical ODE solver [16], for example a Runge-Kutta scheme [42].

\subsection{SBP operators in higher dimensions}

One-dimensional SBP operators can be extended to several spatial dimensions by using tensor products [49], which is illustrated in the two-dimensional case below. The numerical schemes used in Articles I-VI are based on this approach.

To start, recall the Kronecker product [20], which for two matrices $A$ and $B$ are defined as

$$
A \otimes B=\left(\begin{array}{ccc}
a_{11} B & \ldots & a_{1 n} B \\
\vdots & & \vdots \\
a_{m 1} B & \ldots & a_{m n} B
\end{array}\right) .
$$

The Kronecker product satisfies

i) $(A \otimes B)(C \otimes D)=A C \otimes B D$, given that $A C$ and $B D$ are defined.

ii) $(A \otimes B)^{\top}=A^{\top} \otimes B^{\top}$. 


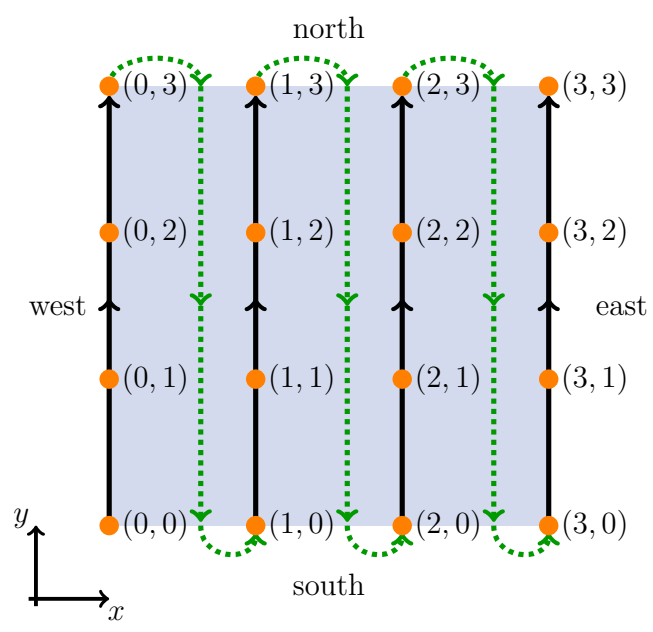

Figure 3.1: Column-wise orientation of a two-dimensional grid. The orange dots represent the grid points and the arrows show the orientation.

iii) $(A \otimes B)^{-1}=A^{-1} \otimes B^{-1}$, provided that $A$ and $B$ are invertible.

To obtain a two-dimensional discrete spatial domain, $\Omega=[0,1] \times[0,1]$ is discretized using $N+1$ and $M+1$ equidistant grid points $\left(x_{i}, y_{j}\right)$ given by $x_{i}=i / N$, $i=0, \ldots N$ and $y_{j}=j / M j=0, \ldots M$. A scalar function $f(x, y)$ defined on $\Omega$ is therefore represented on the grid by $\boldsymbol{f}=\left(f_{00}, \ldots, f_{0 M}, \ldots, f_{N 0}, \ldots f_{N M}\right)^{\top}$, where $f_{i j}=f\left(x_{i}, y_{j}\right)$. The vector $\boldsymbol{f}$ is oriented column-wise, as shown in Figure 3.1.

Remark 2. In the upcoming derivation of the two-dimensional SBP operators, the notations $(\cdot)_{x}$ and $(\cdot)_{y}$ are slightly abused. Capitalization of letters refers to matrices and $D_{x}, P_{x}, B_{x}, D_{y}, P_{y}, B_{y}$ are used to assign the specific spatial directions. For lower-case letters, a notation such as $\boldsymbol{f}_{x}$ refers to a partial derivative evaluated on the grid.

Let $\boldsymbol{D}_{\boldsymbol{x}}=D_{x} \otimes I_{M+1}$ and $\boldsymbol{D}_{\boldsymbol{y}}=I_{N+1} \otimes D_{y}$, where $D_{x}=P_{x}^{-1} Q_{x}$ and $D_{y}=P_{y}^{-1} Q_{y}$ are the one-dimensional SBP operators in the $x$ - and $y$-direction, respectively. Then, the spatial derivatives are approximated by

$$
\boldsymbol{f}_{x} \approx \boldsymbol{D}_{\boldsymbol{x}} \boldsymbol{f} \text { and } \boldsymbol{f}_{\boldsymbol{y}} \approx \boldsymbol{D}_{\boldsymbol{y}} \boldsymbol{f},
$$

where $\boldsymbol{f}_{x}, \boldsymbol{f}_{y}$ hold the partial derivatives $f_{x}, f_{y}$ projected onto the grid. The matrix $\boldsymbol{P}=P_{x} \otimes P_{y}$ is symmetric and positive definite so that $\langle\boldsymbol{v}, \boldsymbol{u}\rangle_{\boldsymbol{P}}=\boldsymbol{v}^{\top} \boldsymbol{P} \boldsymbol{u}$ defines a discrete scalar product and $\|\boldsymbol{f}\|_{\boldsymbol{P}}^{2}=\boldsymbol{f}^{\top} \boldsymbol{P} \boldsymbol{f} \approx \iint_{\Omega} f^{2} \mathrm{~d} x \mathrm{~d} y$ the corresponding discrete norm. Moreover, the two-dimensional SBP operators inherit the SBP property on the two-dimensional grid in the sense that

$$
\boldsymbol{P} \boldsymbol{D}_{\boldsymbol{x}}+\left(\boldsymbol{P} \boldsymbol{D}_{\boldsymbol{x}}\right)^{\top}=\left(Q_{x}+Q_{x}^{\top}\right) \otimes P_{y}=B_{x} \otimes P_{y} .
$$

Hence,

$$
\begin{aligned}
\left\langle\boldsymbol{v}, \boldsymbol{D}_{\boldsymbol{x}} \boldsymbol{u}\right\rangle_{\boldsymbol{P}}+\left\langle\boldsymbol{D}_{\boldsymbol{x}} \boldsymbol{v}, \boldsymbol{u}\right\rangle_{\boldsymbol{P}} & =\boldsymbol{v}^{\top} \boldsymbol{P} \boldsymbol{D}_{\boldsymbol{x}} \boldsymbol{u}+\left(\boldsymbol{D}_{\boldsymbol{x}} \boldsymbol{v}\right)^{\top} \boldsymbol{P} \boldsymbol{u} \\
& =\boldsymbol{v}^{\top} \boldsymbol{P}_{e} \boldsymbol{u}-\boldsymbol{v}^{\top} \boldsymbol{P}_{w} \boldsymbol{u}
\end{aligned}
$$


where $\boldsymbol{P}_{e / w}=E_{(N+1) / 0} \otimes P_{y}$ are the boundary quadratures on the east and west side of $\Omega$, see Figure 3.1. The notation $E_{i}, i \in\{N+1,0\}$ is used to represent a matrix that contains only zeros except for $E_{i, i}=1$. Note that (3.7) mimics the multi-dimensional IBP formula on $\Omega$ :

$$
\left\langle v, u_{x}\right\rangle+\left\langle v_{x}, u\right\rangle=\iint_{\Omega} v u_{x} \mathrm{~d} x \mathrm{~d} y+\iint_{\Omega} v_{x} u \mathrm{~d} x \mathrm{~d} y=\int_{\Gamma_{e}} v u \mathrm{~d} y-\int_{\Gamma_{w}} v u \mathrm{~d} y,
$$

where $\Gamma_{e}$ and $\Gamma_{w}$ are the east and west sides of $\Omega$, respectively. A similar property holds for $\boldsymbol{D}_{\boldsymbol{y}}$. 


\section{Chapter 4}

\section{The incompressible Navier-Stokes equations}

The theory of fluid mechanics is built from conservation laws, where the conserved quantities are mass, momentum and energy. These laws hold for every fluid parcel and can, by the continuum hypothesis, be expressed as system of nonlinear PDEs: The Navier-Stokes equations [50]. Once the equations are solved, a vector field that describes the direction and the magnitude of the velocities is obtained. Other thermodynamic quantities such as the pressure, the density or the temperature can be determined as well. Some example applications are simulating the ocean currents [26], computing the drag force on vehicles [15], and approximating blood flow [38].

Due to the complex nonlinear nature of the equations, it is unknown whether or not a solution exists. The importance of this question can be understood specifically since well-posedness of the INS equations forms one of the Millennium Prize Problems [9]. Hence, numerical methods play an important role. The solutionstrategy used in Articles II-V is presented in this section. It is based on the general procedure in [30], which was later applied to the INS equations in [35].

\subsection{The continuous problem}

The governing equations for a viscous, incompressible fluid (the INS equations) are

$$
\begin{aligned}
\vec{u}_{t}+\vec{u} \cdot \nabla \vec{u}+\nabla p & =\epsilon \Delta \vec{u}, \\
\nabla \cdot \vec{u} & =0,
\end{aligned}
$$

where $\vec{u}=(u, v)^{\top}$ is the velocity field, $p$ is the pressure (divided by the constant density $\rho$ ) and $\epsilon=\nu / \rho$ is the dynamic viscosity [3]. Using matrix-vector notation in a Cartesian coordinate system, (4.1) becomes

$$
\tilde{I} \vec{w}_{t}+A \vec{w}_{x}+B \vec{w}_{y}=\epsilon \tilde{I}\left(\vec{w}_{x x}+\vec{w}_{y y}\right),
$$


where $\vec{w}=(u, v, p)$,

$$
A=\left(\begin{array}{lll}
u & 0 & 1 \\
0 & u & 0 \\
1 & 0 & 0
\end{array}\right), \quad B=\left(\begin{array}{lll}
v & 0 & 0 \\
0 & v & 1 \\
0 & 1 & 0
\end{array}\right), \quad \tilde{I}=\left(\begin{array}{lll}
1 & 0 & 0 \\
0 & 1 & 0 \\
0 & 0 & 0
\end{array}\right)
$$

To obtain a stable discretization with the SBP-SAT technique, we split the nonlinear convective terms by taking an average of the conservative and nonconservative formulations [35] as

$$
A \vec{w}_{x}=\frac{1}{2}\left(A \vec{w}_{x}+(A \vec{w})_{x}-A_{x} \vec{w}\right), \quad B \vec{w}_{y}=\frac{1}{2}\left(B \vec{w}_{y}+(B \vec{w})_{y}-B_{y} \vec{w}\right) .
$$

Since the velocity field is divergence-free, $A_{x}+B_{y}=\tilde{I}\left(u_{x}+v_{y}\right)=0$ and (4.2) is equivalent to

$$
\tilde{I} \vec{w}_{t}+\mathcal{N}(\vec{w})=0
$$

where

$$
\mathcal{N}(\vec{w})=\frac{1}{2}\left[A \vec{w}_{x}+(A \vec{w})_{x}+B \vec{w}_{y}+(B \vec{w})_{y}\right]-\epsilon \tilde{I}\left(\vec{w}_{x x}+\vec{w}_{y y}\right) .
$$

Both $A$ and $B$ are symmetric, hence (4.4) is amenable for the energy method.

Augmented with initial and boundary conditions, we obtain the IBVP of the INS equations

$$
\begin{aligned}
& \tilde{I} \vec{w}_{t}+\mathcal{N}(\vec{w})=0, \quad t>0, \quad(x, y) \in \Omega, \\
& \tilde{I} \vec{w}=\vec{f}(x, y), \quad t=0, \quad(x, y) \in \Omega, \\
& H \vec{w}=\vec{g}, \quad t>0, \quad(x, y) \in \partial \Omega,
\end{aligned}
$$

where $H$ is the boundary operator and $\partial \Omega$ the boundary of the domain $\Omega$.

To derive estimates, the following inner product and norm are introduced for two vector functions $\vec{w}$ and $\vec{v}$ defined on $\Omega$

$$
\langle\vec{w}, \vec{v}\rangle=\int_{\Omega} \vec{w}^{\top} \vec{v} \mathrm{~d} \Omega, \quad\|\vec{w}\|^{2}=\langle\vec{w}, \vec{w}\rangle .
$$

Multiplying the first equation in (4.5) by $2 \vec{w}^{\top}$, integrating over $\Omega$, and using IBP in higher dimensions yields

$$
\frac{d}{d t}\|\vec{w}\|_{\tilde{I}}^{2}+2 \epsilon\|\nabla \vec{w}\|_{\tilde{I}}^{2}=B T
$$

In (4.6), $\|\vec{w}\|_{\tilde{I}}^{2}=\langle\vec{w}, \tilde{I} \vec{w}\rangle$, where $\|\vec{w}\|_{\tilde{I}}$ is a semi-norm, $\|\nabla \vec{w}\|_{\tilde{I}}^{2}=\int_{\Omega} \vec{w}_{x}^{\top} \tilde{I} \vec{w}_{x}+$ $\vec{w}_{y}^{\top} \tilde{I} \vec{w}_{y} \mathrm{~d} \Omega$, and $B T$ are boundary terms. The result below follows directly from (4.6).

Proposition 1. Consider the IBVP (4.5) with homogeneous boundary conditions. If $B T \leq 0$, then $\|\vec{w}\|_{\tilde{I}}^{2}$ and $\|\nabla \vec{w}\|_{\tilde{I}}^{2}$ are bounded.

Proof. If $B T \leq 0$, integrating (4.6) in time yields the result.

The explicit form of multiple versions of $B T$ can be found in [35]. In Articles II-IV, different forms of the boundary conditions $H \vec{w}=\vec{g}$ are investigated with respect to energy boundedness and spectral properties. 


\subsection{The discrete problem}

The continuous equation are discretized in space by the operators presented in Section 3.2. For simplicity, $\Omega$ is chosen to be the unit square, see Figure 3.1. The semi-discrete IVP is

$$
\begin{aligned}
\tilde{\boldsymbol{I}} \overrightarrow{\boldsymbol{w}}_{t}+\mathcal{N}(\overrightarrow{\boldsymbol{w}}) & =\mathcal{S}(\overrightarrow{\boldsymbol{w}}), & & t>0, \\
\tilde{\boldsymbol{I}} \overrightarrow{\boldsymbol{w}} & =\overrightarrow{\boldsymbol{f}}, & & t=0 .
\end{aligned}
$$

In (4.7), $\tilde{\boldsymbol{I}}=\tilde{I} \otimes I_{x} \otimes I_{y}$ and

$$
\begin{aligned}
\mathcal{N}(\overrightarrow{\boldsymbol{w}})= & \frac{1}{2}\left[\boldsymbol{A}\left(I_{3} \otimes \boldsymbol{D}_{\boldsymbol{x}}\right) \overrightarrow{\boldsymbol{w}}+\left(I_{3} \otimes \boldsymbol{D}_{\boldsymbol{x}}\right)(\boldsymbol{A} \overrightarrow{\boldsymbol{w}})\right. \\
& \left.+\boldsymbol{B}\left(I_{3} \otimes \boldsymbol{D}_{\boldsymbol{y}}\right) \overrightarrow{\boldsymbol{w}}+\left(I_{3} \otimes \boldsymbol{D}_{\boldsymbol{y}}\right)(\boldsymbol{B} \overrightarrow{\boldsymbol{w}})\right] \\
& -\epsilon\left[\tilde{I} \otimes\left(\boldsymbol{D}_{\boldsymbol{x}}^{2}+\boldsymbol{D}_{\boldsymbol{y}}^{2}\right)\right] \overrightarrow{\boldsymbol{w}}
\end{aligned}
$$

The penalty term $\mathcal{S}(\overrightarrow{\boldsymbol{w}})$ along physical boundaries constitutes four terms (one for each side of the domain) which are of the general form (see Articles III and IV)

$$
\mathcal{S}_{k}=\left(I_{3} \otimes \boldsymbol{P}^{-1}\right) \boldsymbol{\Sigma}_{k}\left(I_{3} \otimes \boldsymbol{P}_{k}\right)\left(\boldsymbol{H}_{k} \overrightarrow{\boldsymbol{w}}-\overrightarrow{\boldsymbol{g}}\right), \quad k \in\{s, e, n, w\} .
$$

In (4.8), $\boldsymbol{H}_{k}$ is the discrete boundary operator determined by the continuous one, and $\boldsymbol{P}_{k}$ is the boundary quadrature (see Section 3.2 ). The penalty matrix $\boldsymbol{\Sigma}_{k}$ is determined from the energy analysis and depends on the specific choice of $\boldsymbol{H}_{k}$, see Articles II-IV for details. The continuous analysis is now mimicked by multiplying the first equation in (4.7) by $2 \overrightarrow{\boldsymbol{w}}^{\top}\left(I_{3} \otimes \boldsymbol{P}\right)$ from the left and using the SBP property, which yields

$$
\frac{d}{d t}\|\overrightarrow{\boldsymbol{w}}\|_{\tilde{I} \otimes \boldsymbol{P}}^{2}+2 \epsilon\|\boldsymbol{\nabla} \overrightarrow{\boldsymbol{w}}\|_{\tilde{I} \otimes \boldsymbol{P}}^{2}=\boldsymbol{B} \boldsymbol{T},
$$

where $\boldsymbol{B} \boldsymbol{T}$ are boundary terms. All terms in (4.9) are explicitly stated in Articles II and III. The semi-discrete version of Proposition 1 is stated next.

Proposition 2. Consider the IVP (4.7) with homogeneous boundary conditions. If $\boldsymbol{B} \boldsymbol{T} \leq 0$, then $\|\overrightarrow{\boldsymbol{w}}\|_{\tilde{I} \otimes \boldsymbol{P}}^{2}$ and $\|\boldsymbol{\nabla} \overrightarrow{\boldsymbol{w}}\|_{\tilde{I} \otimes \boldsymbol{P}}^{2}$ are bounded.

Proof. If $\boldsymbol{B T} \leq 0$, then integrating (4.9) in time yields the result.

\subsection{Numerical experiments}

To evolve (4.7) in time, SBP-in-time [36] was used in Article III and the implicit backward differentiation formula [42] was used in Article IV. In order to verify the implementation, the method of manufactured solutions [44] was used in both articles. This procedure was also used to verify the implementation of the nonlinear Euler equations in Article I. Table 4.1 shows the errors and convergence rates from a convergence study in Article III. The expected convergence orders for SBP21 and SBP84 are 2 and 5 [49], which agrees well with the result in Table 4.1.

The results presented next are obtained using the numerical scheme in Articles III and IV, which approximate the solution to the INS equations. In Figure 4.1, the 


\begin{tabular}{c|cc|cc}
\hline operator & \multicolumn{2}{|c|}{ SBP21 } & \multicolumn{2}{c}{ SBP84 } \\
\hline N,M & $e_{2}$ & $r_{2}$ & $e_{5}$ & $r_{5}$ \\
\hline 20 & $1.28 \mathrm{e}-02$ & - & $9.23 \mathrm{e}-04$ & - \\
40 & $3.00 \mathrm{e}-03$ & 2.01 & $5.15 \mathrm{e}-05$ & 4.01 \\
60 & $1.31 \mathrm{e}-03$ & 2.00 & $7.44 \mathrm{e}-06$ & 4.67 \\
80 & $7.31 \mathrm{e}-04$ & 2.00 & $1.82 \mathrm{e}-06$ & 4.82 \\
100 & $4.66 \mathrm{e}-04$ & 2.00 & $6.05 \mathrm{e}-07$ & 4.88 \\
\hline Theoretical & & 2 & & 5
\end{tabular}

Table 4.1: Errors $\left(e_{2}\right.$ and $\left.e_{5}\right)$ and convergence rates $\left(r_{2}\right.$ and $\left.r_{5}\right)$.
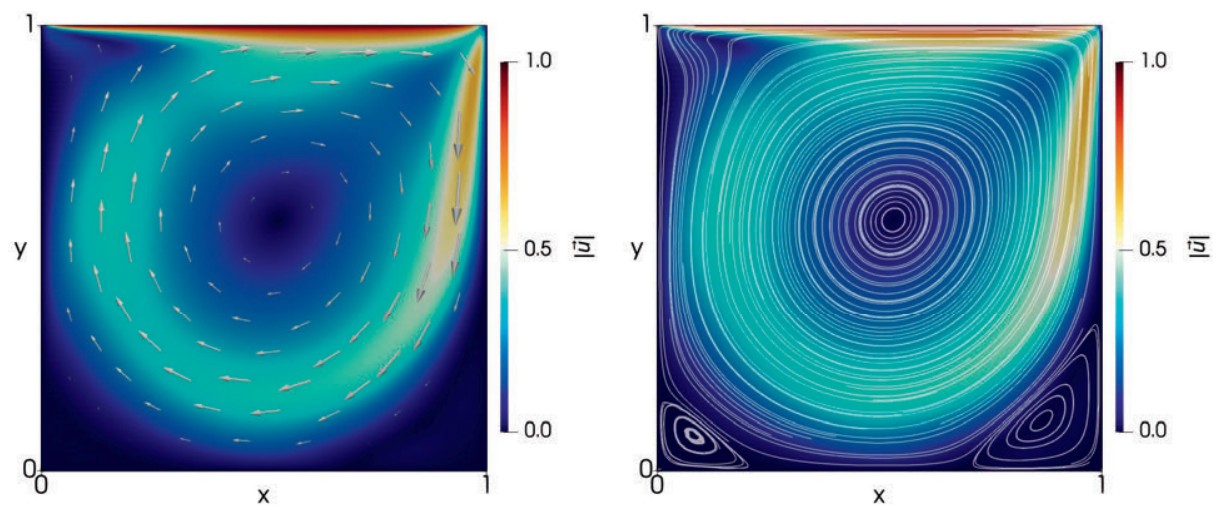

Figure 4.1: Velocity field of the lid-driven cavity flow for $\epsilon=10^{-3}$. All sides except for the top boundary are sold walls. At the top, $(u, v)=(1,0)$ is imposed. The arrows in the left panel show the direction of the velocity field and the solid lines in the right panel are streamlines.

lid-driven cavity flow [14] is considered for $\epsilon=10^{-3}$ on a grid containing $150 \times 150$ grid points. This is a steady-state problem, where the left-hand side, bottom and right-hand side are solid walls. The top is moving with the speed $(u, v)=(1,0)$.

Another example is shown in Figure 4.2, where the SBP operators developed in [1] has been used in combination with the numerical scheme in Articles III and IV. These operators encapsulate the metric terms so that also curved geometries can be handled. The upper and lower boundaries are curved walls, and the fluid is moving from the left to the right. Hence, the west side of the domain is the inflow boundary and the east side is the outflow boundary. As time proceeds, the flow is "squeezed" between the narrowing walls near the center of the domain, which produces a jet. Figure 4.2 shows snapshots at $t=0.5$ and $t=1$ from the time-dependent simulation for $\epsilon=10^{-3}$ on a grid containing $120 \times 120$ grid points. The bottom panel of Figure 4.2 shows a coarse version of the underlying grid with $20 \times 20$ grid points. 

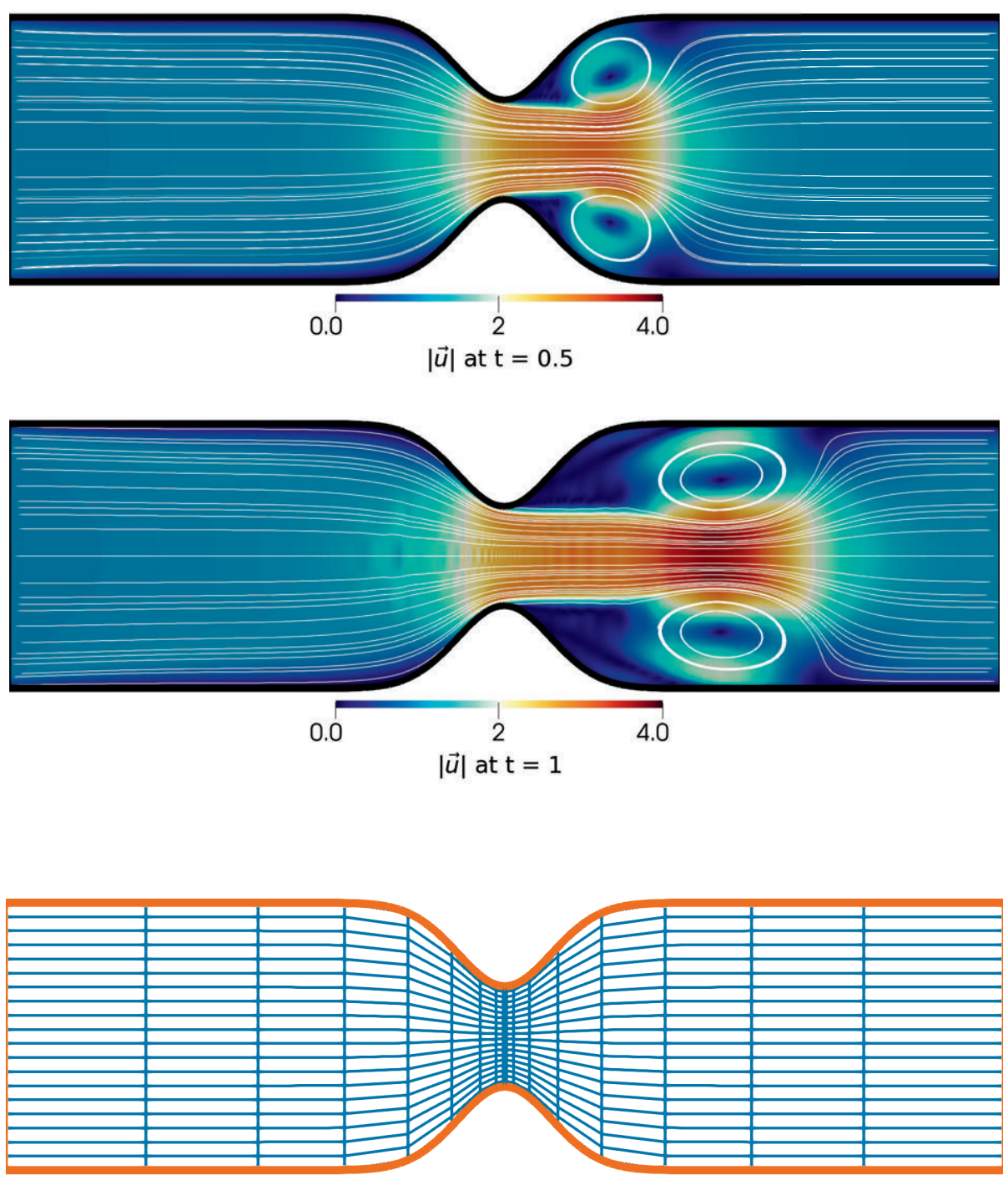

Figure 4.2: Snapshots at $t=0.5$ and $t=1$ of the velocity field during a timedependent simulation on a curved domain. The fluid, moving from the left to the right, is squeezed between the narrow walls of the domain, which leads to a jet. The overlay of white lines are streamlines and the color represents the magnitude of the velocity field. The lowest panel shows a coarse version of the underlying computational grid. 



\section{Chapter 5}

\section{Spectral analysis}

In most numerical calculations, the computational domain must be truncated, which leads to artificial boundaries. At these artificial boundaries, experimental data is imposed via open boundary conditions. The form of the boundary conditions affects the behavior of the solution, which is further studied in Articles I-III.

Steady-state problems are an important subclass of PDEs. There are multiple acceleration-enhancing techniques to improve the convergence rate to steady state such as local time stepping [39] and multi grid [18]. In this section, normal mode analysis [17] is used to estimate the convergence rate to steady state for different sets of open boundary conditions for the INS equations. This procedure has been used in several studies, see for example [28, 7], and the results in this section are found in Article III. A similar procedure is also used in Article I for the compressible Euler equations and Article II for the spatial operator of the INS equations.

To use normal mode analysis, the original nonlinear problem must be linearized and periodicity must be assumed for multi-dimensional problems [17]. Hence, in the upcoming section, $\bar{A}=A(\bar{u}), \bar{B}=B(\bar{v})$ and $\bar{H}=H(\bar{w})$. The solution has been decomposed into a constant mean $\bar{w}=(\bar{u}, \bar{v}, \bar{p})^{\top}$ and a small perturbation $W=\left(u^{\prime}, v^{\prime}, p^{\prime}\right)^{\top}$ so that $\vec{w}=\bar{w}+W$. Furthermore, periodicity is assumed in the $y$-direction. The linear IBVP of the INS equations is

$$
\begin{aligned}
& \tilde{I} W_{t}+\bar{A} W_{x}+\bar{B} W_{y}=\epsilon \tilde{I}\left(W_{x x}+W_{y y}\right), \quad t>0, \quad(x, y) \in \Omega, \\
& \tilde{I} W=\vec{f}(x, y), \quad t=0, \quad(x, y) \in \Omega, \\
& \bar{H}_{0} W=0, \quad t>0, \quad(x, y) \in \Gamma_{0}, \\
& \bar{H}_{1} W=0, \quad t>0, \quad(x, y) \in \Gamma_{1},
\end{aligned}
$$

where $\Omega=[0,1] \times[-\pi, \pi]$ and $\Gamma_{0}=\{(x, y) \in \Omega: x=0\}$ and $\Gamma_{1}=\{(x, y) \in \Omega$ : $x=1\}$ are its west and east sides, respectively. The linear operators $\bar{H}_{0}$ and $\bar{H}_{1}$ impose boundary condition and are at this stage not specified. For the upcoming analysis, it is assumed that the main flow is going in the positive $x$-direction, i.e. $\bar{u}>0$, which means that $\Gamma_{0}$ is an inflow boundary and $\Gamma_{1}$ is an outflow boundary, see Figure 5.1. 


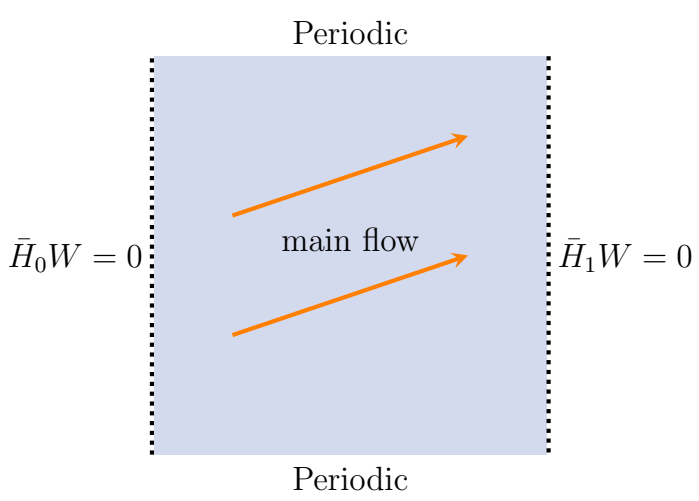

Figure 5.1: The setup of the IBVP (5.1).

\subsection{Fourier analysis}

Consider the fully periodic IVP (the Cauchy problem) of (5.1) on the domain $\Omega=[-\pi, \pi]^{2}$ and apply the Fourier expansion in both spatial directions. The solution becomes

$$
W(t, x, y)=\sum_{i=-\infty}^{\infty} \tilde{W}_{i}(t, \kappa, \omega)=\sum_{\kappa, \omega=-\infty}^{\infty} V(t, \kappa, \omega) e^{i \kappa x} e^{i \omega y} .
$$

In $(5.2), \tilde{W}_{i}(t, \kappa, \omega)$ is one mode, $V(t, \kappa, \omega)$ are the Fourier coefficients and $\kappa, \omega$ are integers. Inserting (5.2) into the first equation of (5.1) leads to

$$
\tilde{I} V_{t}+\left[i \kappa \bar{A}+i \omega \bar{B}+\epsilon\left(\kappa^{2}+\omega^{2}\right) \tilde{I}\right] V=0
$$

for each $\kappa, \omega$. The ansatz $V=\psi e^{s t}$ yields

$$
\underbrace{\left[s \tilde{I}+i \kappa \bar{A}+i \omega \bar{B}+\epsilon\left(\kappa^{2}+\omega^{2}\right) \tilde{I}\right]}_{E(s)} \psi=0 .
$$

For non-trivial solutions $\psi$, it is required that $\operatorname{det}(E(s))=0$ holds, which gives

$$
s^{*}=-i(\kappa \bar{u}+\omega \bar{v})-\epsilon\left(\kappa^{2}+\omega^{2}\right) .
$$

The set $s^{*}$ in (5.3) defines the spectrum [17] of the IVP.

\subsection{Normal mode analysis}

To show the effect of open boundary conditions in the $x$-direction, the periodicity in the $y$-direction is kept and a Fourier expansion is applied in that direction. The first equation in (5.1) becomes

$$
\tilde{I} V_{t}+\bar{A} V_{x}+\left(i \omega \bar{B}+\epsilon \omega^{2} \tilde{I}\right) V=\epsilon \tilde{I} V_{x x} .
$$




\begin{tabular}{c|c|c}
\hline & Inflow conditions & Outflow conditions \\
\hline \hline Set 1 & Characteristic & Characteristic \\
\hline Set 2 & Dirichlet & Natural \\
\hline Set 3 & Stabilized Natural & Stabilized Natural \\
\hline Set 4 & Periodic & Periodic \\
\hline Set 5 & $u=g_{1}$ & Natural and $v=g_{2}$ \\
\hline Set 6 & Modified stabilized natural & Natural \\
\hline
\end{tabular}

Table 5.1: Description of the different sets of boundary conditions.

Next, the ansatz $V=\psi^{s t+\kappa x / \epsilon}$ is used and leads to the Laplace-transformed version of (5.4)

$$
\underbrace{\left[\left(s \epsilon+(\epsilon \omega)^{2}-\kappa^{2}\right) \tilde{I}+\kappa \bar{A}+i(\epsilon \omega) \bar{B}\right]}_{C(s, \kappa, \omega)} \psi=0 .
$$

For non-trivial solutions, $\operatorname{det}(C(s, \kappa, \omega))=0$ must hold, which is solved for $\kappa=$ $\kappa(s)$. In the absence of repeated roots, each mode is of the form

$$
\tilde{W}(s, x, \omega)=\sum_{j=1}^{4} \sigma_{j} \psi_{j} e^{s t+\kappa_{j} x / \epsilon}=\Psi X(x) \vec{\sigma} e^{s t},
$$

where $\psi_{j}$ is the generalized eigenvector for a specific $\kappa_{j}$. Inserting (5.5) into the boundary conditions yields for every $\omega$

$$
E(s) \vec{\sigma}=\left(\begin{array}{c}
\bar{H}_{0} \Psi X(0) \\
\bar{H}_{1} \Psi X(1)
\end{array}\right) \vec{\sigma}=0
$$

where $E(s)$ is a $4 \times 4$ matrix with complex entries (two conditions at the inflow and outflow boundary, respectively).

A non-trivial solution to (5.6) only exists if $E(s)$ is singular. The spectrum of (5.1) is given by [17]

$$
s^{*}=\{s \in \mathbb{C}: \operatorname{det}(E(s))=0\} .
$$

The set $s^{*}$ in (5.7) is found by a numerical root-finding algorithm, such as the Secant method [42]. This approach is used in Articles I-III. Note the difference between (5.3) and (5.7), which is caused by truncating the domain and imposing boundary conditions.

Figure 5.2 shows the spectrum for six different sets of boundary conditions, which are briefly described in Table 5.1. Details are found in Article III. Sets 1-3 as well as the fully periodic case Set 4 satisfy Proposition 1, while Set 5 and Set 6 do not lead to energy bounds and result in ill-posed problems. For Sets 1-4, for which the spectrum resides in the right half of the complex plane, the perturbation $W$ only consists of decaying modes and will therefore decay with time. 

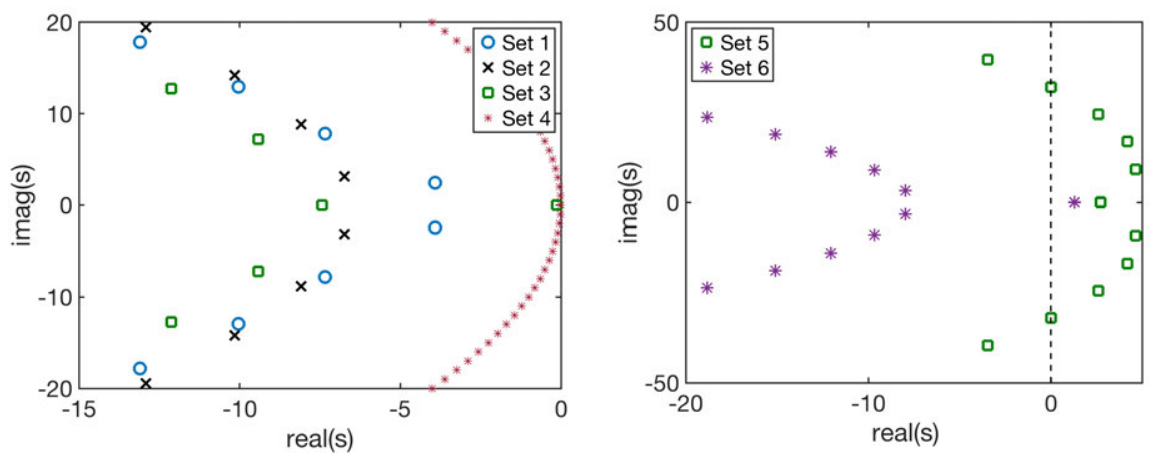

Figure 5.2: The rightmost part of the continuous spectra. Left: Four cases where the spectrum fully resides in the right half of the complex plane. Right: Two sets of boundary conditions that lead to an ill-posed problem is shown.

\subsection{The discrete spectrum}

To compute the discrete spectrum, (5.1) is Fourier-transformed in the $y$-direction and then discretized in the $x$-direction, which yields

$$
\left(\tilde{I} \otimes I_{N+1}\right) \boldsymbol{V}_{t}+\boldsymbol{N} \boldsymbol{V}=0
$$

In (5.8), $\boldsymbol{N}$ is the discrete spatial operator, including the boundary treatment. Inserting the ansatz $\boldsymbol{V}=\boldsymbol{\psi} e^{s t}$, we obtain

$$
\left[s\left(\tilde{I} \otimes I_{N+1}\right)+\boldsymbol{N}\right] \boldsymbol{\psi}=0 .
$$

The discrete spectrum is defined as $s_{D}^{*}=\left\{s \in \mathbb{C}: \operatorname{det}\left(s\left(\tilde{I} \otimes I_{N+1}\right)+\boldsymbol{N}\right)=0\right\}$.

Figure 5.3 shows the discrete spectrum in comparison to its continuous analogue for a low-order operator (SBP21) and a high-order operator (SBP84) [49]. The spectrum of the high-order SBP operator approximates the continuous spectrum much better. The advantage of high-order operators in this context are further discussed in Articles I-III.

\subsection{The convergence rate to steady state}

Let $\eta_{C}^{*}$ denote the maximum real part of the continuous spectrum and $\eta_{D}^{*}$ the corresponding value for the discrete spectrum. Then, for a numerical simulation initialized by a small perturbation from the constant background state, i.e. $\vec{w}=$ $\bar{w}+W$, it will be that $W \rightarrow 0$ at a rate proportional to the discrete decay rate $e^{\eta_{D}^{*} t}$. This discrete decay rate should converge to the true decay rate $e^{\eta_{C}^{*} t}$ under appropriate grid refinement. The results presented next are found in Article III. In Article I, similar experiments are performed for the compressible Euler equations. Periodic boundary conditions are imposed in the $y$-direction and each of the Sets 

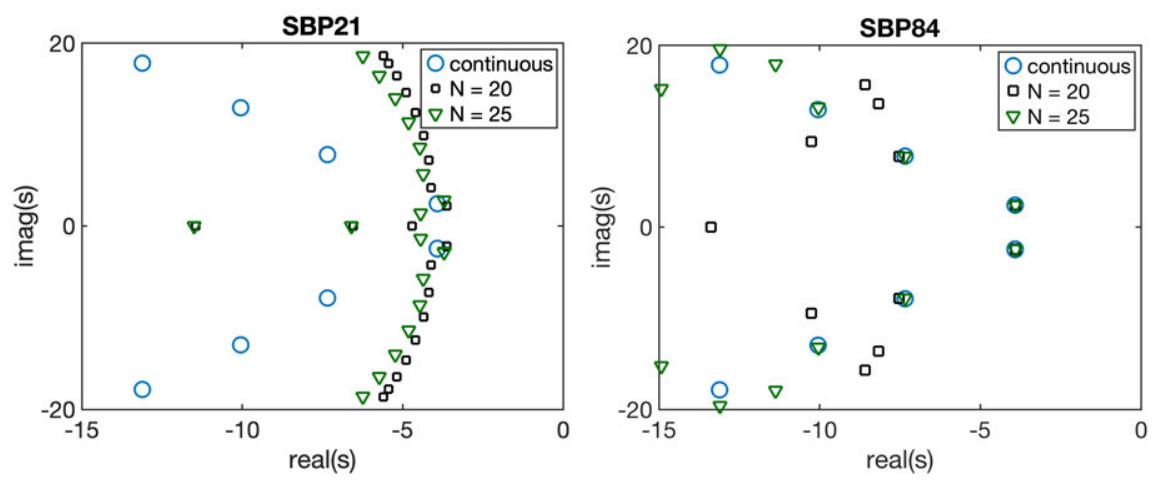

Figure 5.3: Comparison between the discrete and continuous spectra. Note the poor approximations produced by the SBP21 operator compared to SBP84.
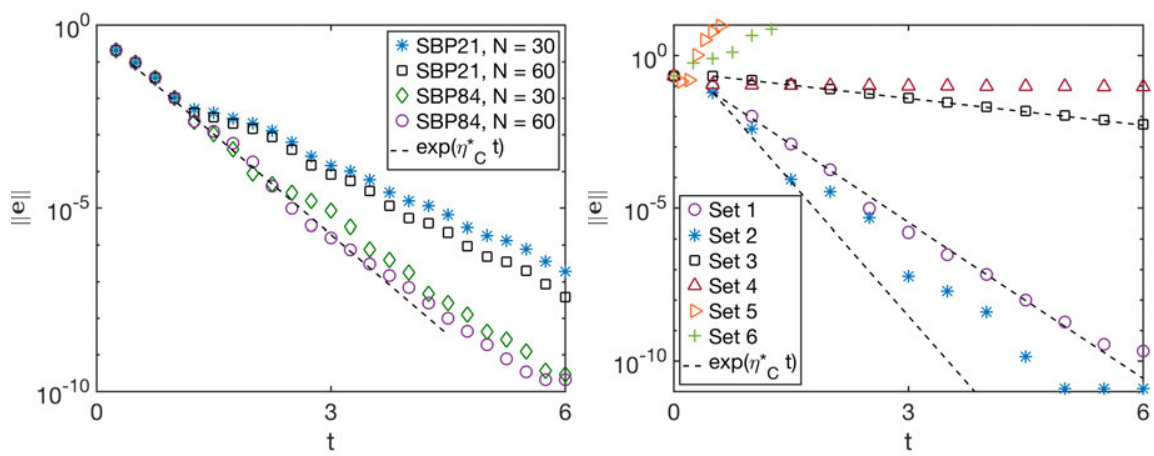

Figure 5.4: Left: Comparison between the discrete and and theoretical decay rate (dashed line) for Set 1. Right: Decay rate for steady-state computations for all sets of boundary conditions. The theoretical decay rates for Sets 1-3 are included for comparison. The solution grows in time for Set 5 and Set 6 .

1-6 are imposed in the $x$-direction. SBP-in-time has been used to evolve the system in time. The error, $\|\boldsymbol{e}\|$, is the norm of the point-wise deviation of $\vec{w}$ from the constant background state $\bar{w}$, see Article III for further details. The left panel of Figure 5.4 shows the convergence rate for the imposition of Set 1 . The decay rate of the high-order operator converges towards the continuous one faster compared to the low-order operator.

In the right panel of Figure 5.4, all six sets of boundary conditions are considered. The estimated decay rates for Sets 1-3 agree well with the numerical results. Set 5 and Set 6 diverge exponentially, which is also in agreement with the theoretical analysis. 



\section{Chapter 6}

\section{Summary of articles}

A summary of each paper is given below.

\subsection{Article I}

Two commonly used $[4,45]$ boundary conditions for internal flow simulations are considered: The specification of the total temperature and total pressure. In nondimensional form, these two quantities are given by

$$
\begin{array}{ll}
T_{0}=T+\left(u^{2}+v^{2}\right)(\gamma-1) M_{\infty}^{2} / 2, & \text { (Total temperature) } \\
p_{0}=p\left[1+\left(u^{2}+v^{2}\right)(\gamma-1) M_{\infty}^{2} /(2 T)\right]^{\gamma /(\gamma-1)} . & \text { (Total pressure) }
\end{array}
$$

The flow is governed by the compressible Euler equations in two spatial dimensions. It is shown that the specification of $T_{0}=g_{1}, p_{0}=g_{2}$ and the tangential velocity at an inflow boundary leads to a well-posed problem for the linearized equations. Finite-difference SBP operators are used to discretize the equations in space and the linear discretization is proven stable.

Next, the spectrum is computed using normal mode analysis and used to estimate the convergence rate to steady state as described in Section 5.2. The implementation of the nonlinear equations is verified by the method of manufactured solutions and further used to confirm the estimated convergence rates.

\subsection{Article II}

The spatial operator of the INS equations can, for certain boundary conditions, be singular and have a non-trivial null space. This implies that the steady-state problem has a non-unique solution. Special techniques, such as specifying an additional integral condition for the pressure [41], can remove the singularity but complicates the well-posedness/stability analysis.

In Article II, we investigate the impact of boundary conditions on the null space of the spatial operator. An illustrative example describing the workflow and the main result is given below. 


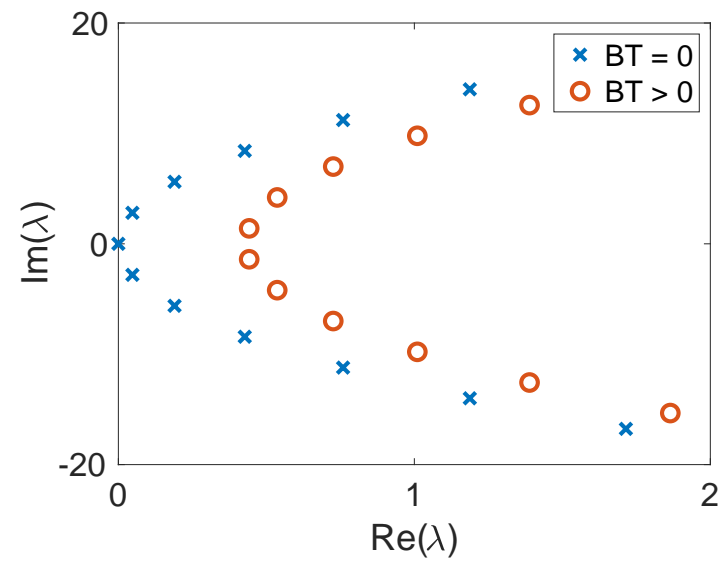

Figure 6.1: Spectrum of the spatial operator. For the boundary conditions leading to $B T=0, \lambda=0$ is an eigenvalue. For the case $B T>0, \lambda=0$ is not an eigenvalue and the spatial operator is non-singular.

Consider the eigenvalue problem of the spatial operator in (5.8)

$$
\boldsymbol{N} \boldsymbol{V}=\lambda \boldsymbol{V}
$$

To determine if $\lambda=0$ is an eigenvalue, we multiply (6.1) by $\boldsymbol{V}^{*}\left(I_{3} \otimes P_{x}\right)$ (where $\boldsymbol{V}^{*}$ denotes the complex conjugate to $\boldsymbol{V}$ ) from the left, use the SBP properties of the difference operators, and get

$$
B T+2 \epsilon\left\|\left(I_{3} \otimes D_{x}\right) \boldsymbol{V}\right\|_{\tilde{I} \otimes P_{x}}^{2}+2 \epsilon \omega^{2}\|\boldsymbol{V}\|_{\tilde{I} \otimes P_{x}}^{2}=2 R e(\lambda)\|\boldsymbol{V}\|_{I_{3} \otimes P_{x}}^{2} .
$$

If $B T>0$ for all $\boldsymbol{V} \neq 0$, then $\operatorname{Re}(\lambda)>0$ and $\boldsymbol{N}$ is non-singular. However, if $B T=0$ for $\boldsymbol{V} \neq 0$, then for $\omega=0, \lambda=0$ is a possible eigenvalue. Figure 6.1 shows two different sets of boundary conditions: One leads to $B T=0$ and the other one results in $B T>0$. For the case corresponding to $B T=0, \lambda=0$ is an eigenvalue. The main result of Article II is that the null space can be removed by specifying appropriate boundary conditions.

\subsection{Article III}

In Article III, different sets of open boundary conditions for the INS equations are considered. The specific form and position of the conditions affect the decay rate and dispersion properties of the solution. By using normal mode analysis, see Section 5.2, we determine the spectra, extract the decay rate in time and investigate the dispersion relation. In contrast to an infinite domain, where only diffusion affects the decay, it is shown that the propagation speed also influence the convergence rate to steady state for a bounded domain. 


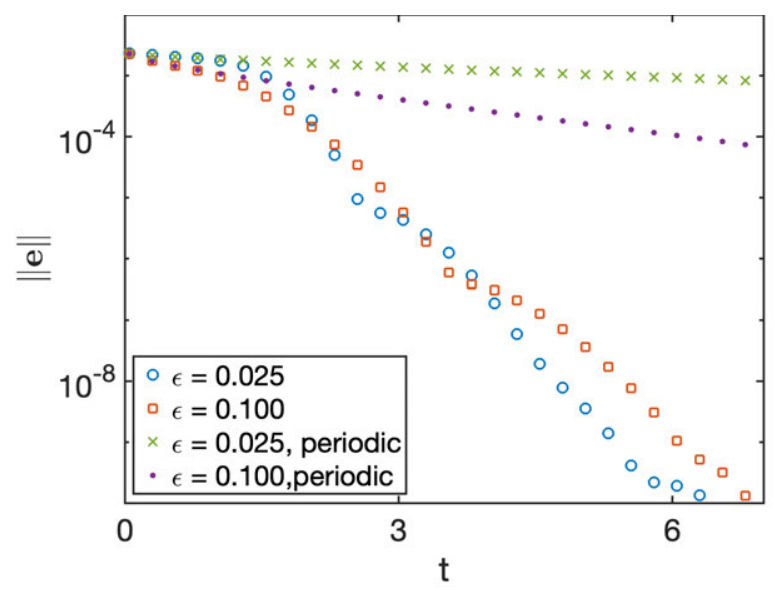

Figure 6.2: Decay rate to steady state for an infinite domain (simulated by imposing periodic boundary conditions) and a bounded domain.

The equations are discretized in space by using the SBP-SAT technique and all findings in the continuous setting carry over to the discrete formulation. It is highlighted that high-order schemes are necessary for a correct description of time-dependent phenomena to avoid excessively fine meshes. In Figure 6.2, the convergence rate to steady state of an infinite domain (simulated by imposing periodic boundary conditions) is shown and compared to the same case on a bounded domain. The solution on the bounded domain vanish much faster when it reaches the boundary.

\subsection{Article IV}

Close to a solid wall, fluids may generate steep gradients which require a fine mesh for an accurate prediction of a turbulent boundary layer. At a no-slip wall, $u=v=0$ holds and the boundary terms in (4.6) vanish. This, however, leads to costly computations. To bypass the fine mesh requirement, wall models of the generic mean flow form

$$
u^{+}=h\left(y^{+}\right)
$$

are frequently used. In (6.2), $u^{+}$is the non-dimensional tangential velocity close to the surface and $y^{+}$is the non-dimensional distance to the wall. The wall model (6.2) is used to produce data for the tangential stress, $\tau_{w}$, which is imposed at the wall together with the no-penetration condition $v=0$. The boundary terms in (4.6) then become

$$
B T=-\int_{\Gamma} 2 u \epsilon u_{y} \mathrm{~d} \Gamma=-\int_{\Gamma} 2 u \tau_{w} \mathrm{~d} \Gamma .
$$

An energy bound is obtained if $u$ at the wall and $\tau_{w}$ with data from the wall model have the same sign, which is not necessarily true $[46,25]$. Hence, instabilities may 
be triggered, leading to an ill-posed problem.

Two strategies were suggested to improve coarse-grid computations. The first procedure uses the classical log-law [40]

$$
u^{+}=\frac{1}{\kappa} \ln \left(y^{+}\right)+C,
$$

where $\kappa$ and $C$ are constants. From (6.4), one can derive the relation

$$
u(y)=K(y) u_{y}, \quad \text { where } K(y)>0,
$$

which can be used as an off-wall boundary condition. Combining (6.3) and (6.5) yields

$$
B T=-\int_{\Gamma} 2 u \epsilon u_{y} \mathrm{~d} \Gamma=-\int_{\Gamma} 2 \epsilon u^{2} / K(y) \mathrm{d} \Gamma
$$

and no instabilities can occur.

The second procedure is to combine the multiple penalty term (MPT) technique with data from (6.4). Additional penalty terms are added on inside the computational domain (i.e. not at the boundaries). This procedure was developed in [31], where it was used to increase the convergence rate to steady state. It has also been used on linear problems to control error growth [34] and to aid coarsegrid computations [32]. In Article IV, we use the MPT technique together with data from the wall model to improve the coarse-grid results for the nonlinear INS equations.

\subsection{Article V}

Single-block SBP operators are combined in order to obtain an operator that inherits the SBP property on the multi-block domain. The interfaces between the blocks are treated with inner-product-preserving (IPP) interpolation operators [27]. The multi-block formulation enables a clean description of the numerical scheme, which is beneficial when it comes to the numerical implementation procedure. Furthermore, the stability analysis on a multi-block domain is simplified, since no problem-specific interface conditions must be derived and implemented.

The derivation of the encapsulated operators can be done by considering the advection equation $u_{t}+u_{x}=0$. On a two-dimensional, single-block domain $\Omega$, the semi-discrete advection equation is written as

$$
\boldsymbol{u}_{t}+\boldsymbol{D}_{\boldsymbol{x}} \boldsymbol{u}=0
$$

The treatment of the physical boundary conditions are ignored, since numerical interfaces are in focus. The SBP operator satisfies

$$
\boldsymbol{D}_{\boldsymbol{x}}=\boldsymbol{P}^{-1} \boldsymbol{Q}_{x}, \quad \boldsymbol{Q}_{x}+\boldsymbol{Q}_{x}^{\top}=E^{\top} P N_{x} E,
$$

where $P$ and $N_{x}$ are diagonal matrices containing the quadrature weights at the boundary and the $x$-components of the outward-pointing unit normal. Furthermore, the matrix $E$ is a restriction operator from the volume mesh to the surface 

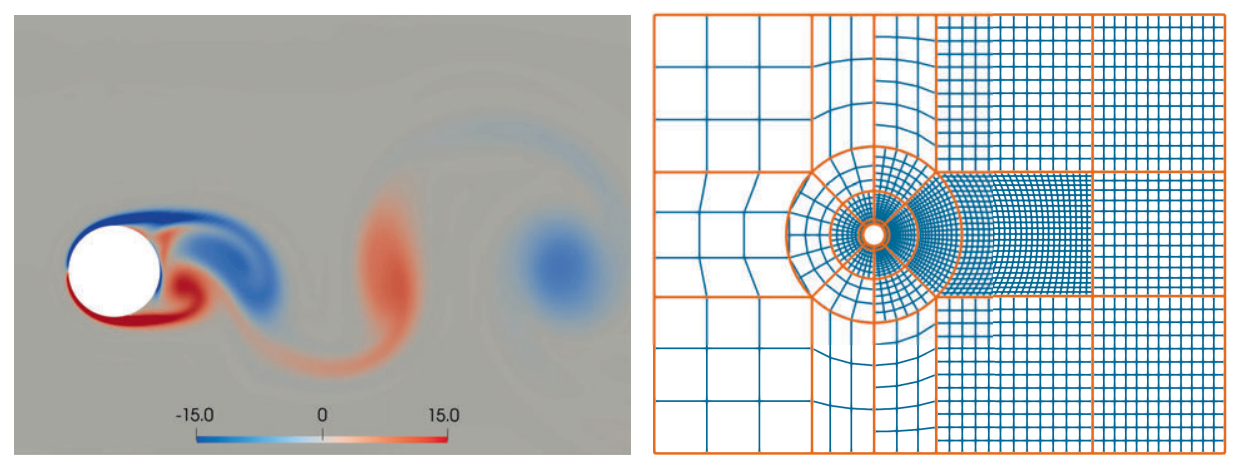

Figure 6.3: Vorticity plot for the INS equations behind a cylinder and a coarse version of the underlying multi-block grid.

mesh. For any vector $\boldsymbol{\Phi}$ of the same size as the volume mesh, $\phi=E \boldsymbol{\Phi}$ contains only the values of $\boldsymbol{\Phi}$ at the surface mesh.

Next, $\Omega$ is split into two parts $\Omega_{L}$ and $\Omega_{R}$ with associated SBP operators $\boldsymbol{D}_{\boldsymbol{x} \boldsymbol{L}}$ and $\boldsymbol{D}_{\boldsymbol{x} \boldsymbol{R}}$. Hence, (6.7) becomes

$$
\begin{aligned}
\boldsymbol{Q}_{\boldsymbol{x} \boldsymbol{L}}+\boldsymbol{Q}_{\boldsymbol{x} \boldsymbol{L}}^{\top} & =\left(E_{L}^{i}\right)^{\top} P_{L}^{i} N_{x L}^{i} E_{L}^{i}+\left(E_{L}^{b}\right)^{\top} P_{L}^{b} N_{x L}^{b} E_{L}^{b}, \\
\boldsymbol{Q}_{\boldsymbol{x} \boldsymbol{R}}+\boldsymbol{Q}_{\boldsymbol{x} \boldsymbol{R}}^{\top} & =\left(E_{R}^{i}\right)^{\top} P_{R}^{i} N_{x R}^{i} E_{R}^{i}+\left(E_{R}^{b}\right)^{\top} P_{R}^{b} N_{x R}^{b} E_{R}^{b}
\end{aligned}
$$

where the superscripts $i$ and $b$ indicates the interface and the physical boundary, respectively.

The advection equation is now discretized in space using a combination of SBP operators and interpolation operators, which results in

$$
\begin{aligned}
\left(\boldsymbol{u}_{L}\right)_{t}+\boldsymbol{D}_{\boldsymbol{x} \boldsymbol{L}} \boldsymbol{u} & =S A T_{L}, \\
\left(\boldsymbol{u}_{R}\right)_{t}+\boldsymbol{D}_{\boldsymbol{x} \boldsymbol{R}} \boldsymbol{u} & =S A T_{R} .
\end{aligned}
$$

In (6.8), $S A T_{L}$ and $S A T_{R}$ contains the interface treatment that uses IPP interpolation operators to communicate values between the two blocks. The explicit forms of $S A T_{L}$ and $S A T_{R}$ are found in Article V. It is also shown in Article V that $(6.8)$ can be written as

$$
\left(\begin{array}{l}
\boldsymbol{u}_{L} \\
\boldsymbol{u}_{R}
\end{array}\right)_{t}+\mathcal{D}_{x}\left(\begin{array}{c}
\boldsymbol{u}_{L} \\
\boldsymbol{u}_{R}
\end{array}\right)=0
$$

where $\mathcal{D}_{x}$ satisfies $(6.7)$ on the two-block domain.

The operator $\mathcal{D}_{x}$ approximates a single partial derivative and can be used to discretize general PDE problems. Specifically, the stability analysis in Section 4.2 can be extended to curved multi-block domains with non-conforming interfaces. An example of vortex shedding behind a cylinder using these new operators is shown in Figure 6.3. 


\subsection{Article VI}

Specific quantities of a continuous formulation are in some situations desirable to preserve on a discrete level. Some examples are the kinetic energy [21] or the entropy [12] for compressible flows or demanding a solenoidal magnetic filed in magneto-hydrodynamics [19].

In Article VI, a novel numerical scheme for the simulation of dissipative quantum dynamics is developed. The unit trace, defined by

$$
\int \delta(x-y) \rho \mathrm{d} x \mathrm{~d} y=1,
$$

is preserved on a continuous level. The preservation of the unit-trace enables a probability interpretation of the density matrix and relies on a special case of the product rule. Consider the coordinate transformation $z^{\prime}=x+y$ and $z=x-y$. On a continuous level, the trace is preserved due to the fact that we can freely switch between the two sets of variables $(x, y)$ and $\left(z^{\prime}, z\right)$. More precisely, the following relations hold for the partial derivatives

$$
\frac{\partial}{\partial z^{\prime}}=\frac{1}{2}\left(\frac{\partial}{\partial x}+\frac{\partial}{\partial y}\right), \quad \frac{\partial}{\partial z}=\frac{1}{2}\left(\frac{\partial}{\partial x}-\frac{\partial}{\partial y}\right) .
$$

For the functions $\psi\left(z^{\prime}\right)$ and $\phi(z)$, the following hold

$$
\frac{\partial}{\partial z^{\prime}} \phi \psi=\phi \frac{\partial}{\partial z^{\prime}} \psi, \quad \frac{\partial}{\partial z} \phi \psi=\psi \frac{\partial}{\partial z} \phi .
$$

However, using the standard SBP operators presented in Section 3.2 to define the operators $\partial / \partial z$ and $\partial / \partial z^{\prime}$ by

$$
\boldsymbol{D}_{z^{\prime}}=\frac{1}{2}\left(\boldsymbol{D}_{\boldsymbol{x}}+\boldsymbol{D}_{\boldsymbol{y}}\right), \quad \boldsymbol{D}_{z}=\frac{1}{2}\left(\boldsymbol{D}_{\boldsymbol{x}}-\boldsymbol{D}_{\boldsymbol{y}}\right)
$$

do not satisfy (6.9). A possible cure for the second-order periodic case is to define new SBP operators by averaging shifted operators, i.e.,

$$
\boldsymbol{D}_{\boldsymbol{x}}=\frac{1}{2}\left(D \otimes S_{+}+D \otimes S_{-}\right), \quad \boldsymbol{D}_{\boldsymbol{y}}=\frac{1}{2}\left(S_{+} \otimes D+S_{-} \otimes D\right),
$$

where $D$ is the periodic second-order central difference operator and $S_{+}$and $S_{-}$ are shift operators given by

$$
S_{+}=\left(\begin{array}{cccccc}
0 & 0 & 0 & \ldots & 0 & 1 \\
1 & 0 & 0 & \ldots & 0 & 0 \\
0 & 1 & 0 & \ldots & 0 & 0 \\
\vdots & \vdots & \vdots & \ldots & \vdots & \vdots \\
0 & 0 & 0 & \ldots & 1 & 0
\end{array}\right), \quad S_{-}=S_{+}^{\top} .
$$

It is shown in Article VI that using the operators in (6.11) to construct $\boldsymbol{D}_{z^{\prime}}$ and $\boldsymbol{D}_{z}$ leads to that (6.9) holds in the discrete setting. These operators are used to discretize the Lindblad equations and the solution is shown to preserve the unit trace. 


\section{References}

[1] O. Ålund and J. Nordström. Encapsulated high order difference operators on curvilinear non-conforming grids. Journal of Computational Physics, 385:209224, 2019.

[2] D. N. Arnold, F. Brezzi, B. Cockburn, and L. D. Marini. Unified analysis of discontinuous Galerkin methods for elliptic problems. SIAM journal on numerical analysis, 39(5):1749-1779, 2002.

[3] C. K. Batchelor and G. Batchelor. An introduction to fluid dynamics. Cambridge university press, 2000.

[4] L. Cai, J. Xiao, S. Wang, S. Gao, J. Duan, and J. Mao. Gas-particle flows and erosion characteristic of large capacity dry top gas pressure recovery turbine. Energy, 120:498-506, 2017.

[5] M. H. Carpenter, T. C. Fisher, E. J. Nielsen, and S. H. Frankel. Entropy stable spectral collocation schemes for the Navier-Stokes equations: Discontinuous interfaces. SIAM Journal on Scientific Computing, 36(5):B835-B867, 2014.

[6] M. H. Carpenter, D. Gottlieb, and S. Abarbanel. Time-stable boundary conditions for finite-difference schemes solving hyperbolic systems: Methodology and application to high-order compact schemes. Journal of Computational Physics, 111(2):220-236, 1994.

[7] B. Engquist and B. Gustafsson. Steady state computations for wave propagation problems. Mathematics of Computation, 49(179):39-64, 1987.

[8] L. C. Evans. Partial differential equations, volume 19. American Mathematical Society, 2010.

[9] C. L. Fefferman. Existence and smoothness of the Navier-Stokes equation. The millennium prize problems, 57(67):22, 2006.

[10] D. C. D. R. Fernández, P. D. Boom, and D. W. Zingg. A generalized framework for nodal first derivative summation-by-parts operators. Journal of Computational Physics, 266:214-239, 2014.

[11] D. C. D. R. Fernández, J. E. Hicken, and D. W. Zingg. Review of summationby-parts operators with simultaneous approximation terms for the numerical 
solution of partial differential equations. Computers \& Fluids, 95:171-196, 2014.

[12] T. C. Fisher, M. H. Carpenter, J. Nordström, N. K. Yamaleev, and C. Swanson. Discretely conservative finite-difference formulations for nonlinear conservation laws in split form: Theory and boundary conditions. Journal of Computational Physics, 234:353-375, 2013.

[13] G. J. Gassner. A skew-symmetric discontinuous Galerkin spectral element discretization and its relation to SBP-SAT finite difference methods. SIAM Journal on Scientific Computing, 35(3):A1233-A1253, 2013.

[14] U. Ghia, K. N. Ghia, and C. Shin. High-Re solutions for incompressible flow using the Navier-Stokes equations and a multigrid method. Journal of computational physics, 48(3):387-411, 1982.

[15] E. Guilmineau. Computational study of flow around a simplified car body. Journal of wind engineering and industrial aerodynamics, 96(6-7):1207-1217, 2008.

[16] B. Gustafsson. High order difference methods for time dependent PDE, volume 38. Springer Science \& Business Media, 2007.

[17] B. Gustafsson, H.-O. Kreiss, and J. Oliger. Time Dependent Problems and Difference Methods. John Wiley \& Sons, Inc., 1995.

[18] W. Hackbusch. Multi-grid methods and applications, volume 4. Springer Science \& Business Media, 2013.

[19] C. Helzel, J. A. Rossmanith, and B. Taetz. An unstaggered constrained transport method for the 3D ideal magnetohydrodynamic equations. Journal of Computational Physics, 230(10):3803-3829, 2011.

[20] R. A. Horn and C. R. Johnson. Topics in matrix analysis. Cambridge university press, 1994.

[21] A. Jameson. Formulation of kinetic energy preserving conservative schemes for gas dynamics and direct numerical simulation of one-dimensional viscous compressible flow in a shock tube using entropy and kinetic energy preserving schemes. Journal of Scientific Computing, 34(2):188-208, 2008.

[22] H.-O. Kreiss and J. Lorenz. Initial-boundary value problems and the NavierStokes equations. SIAM, 2004.

[23] H.-O. Kreiss and G. Scherer. Finite element and finite difference methods for hyperbolic partial differential equations. In Mathematical aspects of finite elements in partial differential equations, pages 195-212. Elsevier, 1974.

[24] H.-O. Kreiss and G. Scherer. On the existence of energy estimates for difference approximations for hyperbolic systems. Technical report, Technical report, Dept. of Scientific Computing, Uppsala University, 1977. 
[25] J. Larsson, S. Kawai, J. Bodart, and I. Bermejo-Moreno. Large eddy simulation with modeled wall-stress: recent progress and future directions. $\mathrm{Me}$ chanical Engineering Reviews, 3(1):15-00418, 2016.

[26] J. Marshall, A. Adcroft, C. Hill, L. Perelman, and C. Heisey. A finite-volume, incompressible Navier Stokes model for studies of the ocean on parallel computers. Journal of Geophysical Research: Oceans, 102(C3):5753-5766, 1997.

[27] K. Mattsson and M. H. Carpenter. Stable and accurate interpolation operators for high-order multiblock finite difference methods. SIAM Journal on Scientific Computing, 32(4):2298-2320, 2010.

[28] J. Nordström. The influence of open boundary conditions on the convergence to steady state for the Navier-Stokes equations. Journal of Computational Physics, 85(1):210-244, 1989.

[29] J. Nordström. Conservative finite difference formulations, variable coefficients, energy estimates and artificial dissipation. Journal of Scientific Computing, $29(3): 375-404,2006$.

[30] J. Nordström. A roadmap to well posed and stable problems in computational physics. Journal of Scientific Computing, 71:365-385, 2017.

[31] J. Nordström, Q. Abbas, B. A. Erickson, and H. Frenander. A flexible boundary procedure for hyperbolic problems: Multiple penalty terms applied in a domain. Communications in Computational Physics, 16(2):345-358, 2014.

[32] J. Nordström and O. Ålund. Neural network enhanced computations on coarse grids. Journal of Computational Physics, 425:109821, 2021.

[33] J. Nordström, K. Forsberg, C. Adamsson, and P. Eliasson. Finite volume methods, unstructured meshes and strict stability for hyperbolic problems. Applied Numerical Mathematics, 45(4):453-473, 2003.

[34] J. Nordström and H. Frenander. On long time error bounds for the wave equation on second order form. Journal of Scientific Computing, 76(3):13271336, 2018.

[35] J. Nordström and C. La Cognata. Energy stable boundary conditions for the nonlinear incompressible Navier-Stokes equations. Mathematics of Computation, 88(316):665-690, 2019.

[36] J. Nordström and T. Lundquist. Summation-by-parts in time. Journal of Computational Physics, 251:487-499, 2013.

[37] J. Nordström and A. A. Ruggiu. On conservation and stability properties for summation-by-parts schemes. Journal of Computational Physics, 344:451464, 2017. 
[38] M. S. Olufsen, C. S. Peskin, W. Y. Kim, E. M. Pedersen, A. Nadim, and J. Larsen. Numerical simulation and experimental validation of blood flow in arteries with structured-tree outflow conditions. Annals of biomedical engineering, 28(11):1281-1299, 2000.

[39] S. Osher and R. Sanders. Numerical approximations to nonlinear conservation laws with locally varying time and space grids. Mathematics of computation, 41(164):321-336, 1983.

[40] S. B. Pope and S. B. Pope. Turbulent flows. Cambridge university press, 2000.

[41] L. Quartapelle and M. Napolitano. Integral conditions for the pressure in the computation of incompressible viscous flows. Journal of Computational Physics, 62(2):340-348, 1986.

[42] A. Quarteroni, R. Sacco, and F. Saleri. Numerical mathematics, volume 37. Springer Science \& Business Media, 2010.

[43] S. S. Rao. Applied numerical methods for engineers and scientists. Prentice Hall Professional Technical Reference, 2001.

[44] P. J. Roache. Code verification by the method of manufactured solutions. J. Fluids Eng., 124(1):4-10, 2002.

[45] A. Romagnoli and R. Martinez-Botas. Performance prediction of a nozzled and nozzleless mixed-flow turbine in steady conditions. International Journal of Mechanical Sciences, 53(8):557-574, 2011.

[46] V. Singh, S. Frankel, and J. Nordström. Impact of wall modeling on kinetic energy stability for the compressible Navier-Stokes equations. Computers $\&$ Fluids, 220:104870, 2021.

[47] W. A. Strauss. Partial differential equations: An introduction. John Wiley \& Sons, 2007.

[48] J. C. Strikwerda. Finite difference schemes and partial differential equations. SIAM, 2004.

[49] M. Svärd and J. Nordström. Review of summation-by-parts schemes for initial-boundary-value problems. Journal of Computational Physics, 268:17$38,2014$.

[50] F. White. Fluid mechanics. McGraw-Hill Education, 2003. 


\section{Papers}

The papers associated with this thesis have been removed for copyright reasons. For more details about these see:

https://doi.org/10.3384/9789179291228 



\section{FACULTY OF SCIENCE AND ENGINEERING}

Linköping Studies in Science and Technology, Dissertation No. 2190, 2022 Department of Mathematics

Linköping University

SE-581 83 Linköping, Sweden

www.liu.se

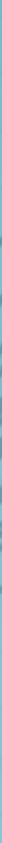

